\title{
A new perspective: imaging the stereochemistry of molecular collisions
}

\author{
F. J. Aoiz ${ }^{a}$, M. Brouard ${ }^{b *}$, S. D. S. Gordon ${ }^{b}$, \\ B. Nichols ${ }^{b}$, S. Stolte ${ }^{c, d, e \ddagger}$ and V. Walpole ${ }^{b}$
}

August 11, 2015

\begin{abstract}
The concept of the steric effect in molecular collisions is central to chemistry. In this Perspective article we review some of the progress made in studying the steric effect in inelastic and reactive collisions involving relatively small isolated atomic and molecular species. We overview the theoretical framework used to quantify the steric effect, and outline some of the key experimental approaches that can be employed to study the dynamics and mechanism of collisions involving oriented and alignment molecules. We illustrate the discussion by highlighting a few recent studies of inelastic and reactive scattering. Finally, we conclude with some reflections on possible future directions of interest.
\end{abstract}

\footnotetext{
${ }^{a}$ Departamento de Química Física, Facultad de Química, Universidad Complutense, 28040 Madrid, Spain

${ }^{b}$ The Department of Chemistry, University of Oxford, The Physical and Theoretical Chemistry Laboratory, South Parks Road, Oxford, OX1 3QZ, United Kingdom

${ }^{c}$ Institute of Atomic and Molecular Physics, Jilin University, Changchun 130012, China

${ }^{d}$ Department of Physics and Astronomy, LaserLaB, Vrije Universiteit, Amsterdam, de Boelelaan 1081, 1081 HV Amsterdam, The Netherlands

${ }^{e}$ Laboratoire Francis Perrin, Bâtiment 522, DRECEM/SPAM/CEA Saclay, 91191 Gif sur Yvette, France

${ }^{*}$ To whom correspondence should be addressed; E-mail: mark.brouard@chem.ox.ac.uk

E-mail addresses: ${ }^{\star}$ S.stolte@vu.nl, ${ }^{\natural}$ aoiz@quim.ucm.es
} 


\section{Introduction}

Most, if not all, chemistry graduates will be aware of the importance of steric effects in influencing the outcome of chemical reactions. Stereochemistry plays a central role in developing stereoselective pathways to new compounds. Stereo-specific effects also lie at the heart of many selective biological processes. In this Perspective article we will consider relatively simple prototype collision systems, isolated in the gas phase, and consider how the reaction or scattering probability varies when one of the reactants is polarized in a well-defined fashion. The motivation for such studies is the insight that they provide about the mechanism of inelastic and reactive collisions, and the opportunity they provide for a detailed and quantitative understanding of the scattering process.

Although steric effects in isolated bimolecular collisions have been the focus of numerous investigations over the last 50 years, ${ }^{1}$ there has been a recent resurgence of interest in the effects of reactant polarization on chemical reactivity, in part heralded by the development of new and sophisticated experimental techniques. Here we report on some of these recent advances in probing and understanding steric effects in isolated atomic and molecular collisions.

Dynamical stereochemistry (or stereodynamics) ${ }^{2}$ is the name given to the field involved in the study of the dependence of reactive or inelastic scattering processes on the polarization of one or more of the reactants. The language employed by 'dynamical stereochemists' is that of vector correlations, and there is no avoiding the use of the language of vector correlations ${ }^{3,4}$ in some of what follows. The term vector correlation indicates that one is interested in quantifying the preferred relationships between two or more vector quantities, which possess both magnitude and direction. Determining a vector correlation between two vectors is thus, in part, a matter of quantifying the angular distribution between them. In addition, one might also wish to measure how the cross section for a given process varies with the angle between two (or more) vector quantities, although in practice that can be a much more challenging task than measuring the angular distribution alone.

When considering the polarization of one vector with respect to another we need to distinguish orientation and alignment. ${ }^{5,6}$ Consider the bond axis of a heteronuclear diatomic, $r$. The bond axis would be oriented with respect to some reference direction, say $z$, if the vector $\boldsymbol{r}$ points either parallel or antiparallel to $z$. The bond axis would be aligned if $\boldsymbol{r}$ is directed either along (i.e. either parallel or antiparallel) or perpendicular to $z$. Experiments that measure the effect of the alignment and orientation of $\boldsymbol{r}$ 
on chemical reactivity probe different, distinctive aspects of the steric effect, and hence of the underlying interaction potential between the colliding partners. We will consider experiments and theory in which either or both of these aspects are quantified.

Let us illustrate further by considering the scattering between a simple atom, A, and a diatomic, BC, both of which we will assume for now are closed shell species. The measurement of a steric effect corresponds to a vector correlation which defines how the reaction or inelastic scattering probability (or cross section) varies with the polarization of the initial bond axis, $r$, or rotational angular momentum, $j$, of BC with respect to some well-defined axis. A sensible choice of reference axis is the initial relative velocity, $\boldsymbol{k}$, of the A + BC collision system. Measuring the integral steric effect therefore involves measuring how the reaction cross section varies with the angle of $r$ with respect to $k$, or the direction of $j$ with respect to $\boldsymbol{k}$. Such measurements would allow the quantifying of the $\boldsymbol{k}-\boldsymbol{r}$ or the $\boldsymbol{k}$ - $\boldsymbol{j}$ vector correlations. Note that the dependence of the cross section on either the $\mathrm{BC}$ bond axis or its angular momentum polarization could be considered. As we will see in Section 2, these two quantities are closely related.

It is also possible to measure how the reactivity or scattering cross section depends both on the reactant polarization and the direction of the final relative velocity, $\boldsymbol{k}^{\prime}$. This is referred to as a differential steric effect. The differential steric effect quantifies a three vector correlation, either $\boldsymbol{k}-\boldsymbol{j}-\boldsymbol{k}^{\prime}$ or $\boldsymbol{k}-\boldsymbol{r}-\boldsymbol{k}^{\prime}$. As will be discussed in Section 3, this is achieved by observing how the angular distribution of the scattered products, or the differential cross section (DCS), varies with reactant bond axis or angular momentum polarization. In the absence of such reactant polarization, the measurement would yield the conventional DCS, which quantifies the two-vector $\boldsymbol{k}$ - $\boldsymbol{k}^{\prime}$ correlation, i.e. the dependence of the reaction cross section on scattering angle, $\theta$. Note that integration of the three vector correlations $\boldsymbol{k}-\boldsymbol{j}$ - $\boldsymbol{k}^{\prime}$ or $\boldsymbol{k}-\boldsymbol{r}-\boldsymbol{k}^{\prime}$ over scattering angle yields the two-vector correlations $\boldsymbol{k}$ - $\boldsymbol{j}$ or $\boldsymbol{k}-\boldsymbol{r}$, respectively. As with the integral steric effect, the motivation for measuring the differential steric effect is to provide greater insight into how chemical reactivity or scattering mechanism varies with angle of approach of the reactants.

As will be clear from the preceding discussion, here we will focus on measurements in which a reactant molecule or its angular momentum is polarized in space. We will not discuss dynamical measurements in which the reactants are unpolarized, and thus isotropically distributed. That is not to say that such dynamical studies, involving unpolarized reactants, cannot provide valuable information about the stereodynamics of a reaction. The recent work by Wester and coworkers on the scattering dynamics of anion-molecule nucleophilic substitution $\left(S_{N} 2\right)$ reactions provides an excellent example of such a study. ${ }^{7}$ 
However, for reasons of space, studies involving unpolarized reactants are beyond the scope of the present review.

This Perspective article is structured as follows. Section 2 provides the theoretical background needed to understand and interpret stereodynamical experiments, whilst Section 3 provides a brief account of methods used to polarize (orient and align) reactant molecules. Section 4 then presents some recent illustrations of the measurement of both the integral and differential steric effects. Finally, Section 5 summarizes the work described in this Perspective, and provides some suggestions for possible future directions of study.

\section{Theoretical background}

The theoretical description of reactant polarization effects makes use of the distinction between extrinsic and intrinsic polarization. ${ }^{8-10}$ The former concerns the various possible experimental schemes for orienting or aligning reactant molecules. It tells us about the rotational angular momentum and molecular bond axis distributions in the asymptotic region, prior to any interaction between the reactants. As such, extrinsic polarization moments are a consequence of external circumstances (the experimental setup) rather than the reaction itself; they have nothing to do with the collision dynamics. There are many different ways to prepare an ensemble of polarized reactant molecules, limited only by experimental feasibility and the experimenter's ingenuity - see Section 3. Although, in principle, both reactants can be polarized, usually only one of them is furnished with a specific anisotropic distribution.

In contrast, the intrinsic polarization distribution describes the collisional process itself. ${ }^{9}$ It contains all the possible information on the dependence of the cross section for the scattering process on the anisotropies of the rotational angular momentum and molecular axis distributions of the reactants. Intrinsic polarization moments are inherent to the collision process and are independent of external circumstances (the experimental setup). From the quantum mechanical (QM) point of view, all of the information is embedded in the scattering matrix, ${ }^{11}$ but this information needs to be manipulated and adapted to address the problem of how the reactant polarization influences the reactivity. From the experimental point of view, the determination of the intrinsic polarization often implies a particular reactant extrinsic preparation, and usually involves performing measurements (e.g., of integral or differential cross sections) in several different experimental arrangements. Ideally, with a set of different reactant preparation schemes 
or geometries it is possible to extract the integral or differential polarization moments of interest.

The mathematical framework to describe the extrinsic and intrinsic polarization is indeed very similar, but care should be exercised to differentiate the meaning of both descriptions.

\subsection{Extrinsic preparation of polarization}

Let us start by introducing the main ingredients for the description of the preparation of the reactant polarization. We consider the case in which the polarized reactant is a closed-shell diatomic molecule in a well-defined rotational state, $j$. Classically, we can define a probability density function (PDF), $P\left(\Theta_{j}, \Phi_{j}\right)$, that gives the probability of preparing the reactant angular momentum in a well-defined laboratory direction $\Theta_{j}, \Phi_{j}$, defined with respect to some laboratory axes, such as the light polarization vector or the direction of an external field. We can express this PDF as an expansion in spherical harmonics

$$
P\left(\Theta_{j}, \Phi_{j}\right)=\sum_{k=0} \sum_{q=-k}^{k} \frac{2 k+1}{4 \pi} A_{q}^{(k)} C_{k q}^{*}\left(\Theta_{j}, \Phi_{j}\right),
$$

where $C_{k q}\left(\Theta_{j}, \Phi_{j}\right)=[4 \pi /(2 k+1)]^{1 / 2} Y_{k q}\left(\Theta_{j}, \Phi_{j}\right)$ are modified spherical harmonics. ${ }^{6}$ This kind of expansion is called multipolar expansion, wherein the spherical harmonics are the multipoles and the expansion coefficients, $A_{q}^{(k)}\left(=[-1]^{q} A_{-q}^{(k) *}\right)$, are the multipole or polarization moments. Their values control the form of the PDF and define the molecular polarization of the rotational angular momentum. The polarization moment with $k=0$ represents the monopole, which is just the specific normalization of the PDF. Successive moments of rank $k=1,2,3, \cdots$ are the dipole, quadrupole, octupole, etc.. Therefore, by polarizing a molecule, one is extrinsically modifying the probability density function into a prepared spatial configuration. Different probability density functions describe the polarization of $j$ and concurrently, as it will be shown, the polarization of $r$.

So far the expansion in Eq. (1) assumes that the direction of $j$ can be sharply defined. However, due to the uncertainty principle, one cannot localize angular momentum vectors as precisely quantum mechanically as one can classically. Additionally, the PDF of Eq. (1) may become negative when truncating the summation to $k=2 j$, the highest possible rank of these parameters. ${ }^{5,6,10}$ 
The correct QM expression for the $P\left(\Theta_{j}, \Phi_{j}\right)$ PDF can be written ${ }^{12,13}$

$$
P\left(\Theta_{j}, \Phi_{j}\right)=\sum_{k=0}^{2 j} \sum_{q=-k}^{k} \frac{2 k+1}{4 \pi} A_{q}^{(k)}\langle j j, k 0 \mid j j\rangle C_{k q}^{*}\left(\Theta_{j}, \Phi_{j}\right) .
$$

The introduction of the $\langle j j, k 0 \mid j j\rangle$ Clebsch-Gordan (CG) coefficient automatically ensures that the PDF cannot be negative at any value of $\Theta_{j}, \Phi_{j}$, as long as the values of the polarization parameters are within their quantum limits.

The polarization of $j$ implies the polarization of the internuclear axis, $\boldsymbol{r}$, and therefore one can be linked to the other. It can be shown that the PDF describing the $\boldsymbol{r}$ polarization can be written as $5,12,13$

$$
P\left(\Theta_{r}, \Phi_{r}\right)=\sum_{k=0}^{2 j} \sum_{q=-k}^{k} \frac{2 k+1}{4 \pi} A_{q}^{(k)}\langle j 0, k 0 \mid j 0\rangle C_{k q}^{*}\left(\Theta_{r}, \Phi_{r}\right) .
$$

In this case, the CG coefficient that appears in Eq. (3), $\langle j 0, k 0 \mid j 0\rangle$, is zero unless $k$ is even. Therefore, the PDF is only sensitive to alignment (even $k$ ) moments. Thus, for a molecule in a well-defined $j$ state and in the absence of an external field, one can prepare an oriented distribution of rotational angular momentum, but only an aligned distribution of the bond axis.

The polarization moments for $q \neq 0$ in the above equations are complex. However, it is easy to transform them into so called real polarization moments using the Hertell-Stoll procedure: ${ }^{14}$

$$
\begin{aligned}
& A_{q+}^{\{k\}}=\frac{\sqrt{2}}{2}\left[(-1)^{q} A_{+q}^{(k)}+A_{-q}^{(k)}\right]=(-1)^{q} \sqrt{2} \operatorname{Re}\left(A_{+q}^{(k)}\right), \quad q>0, \\
& A_{q-}^{\{k\}}=-\frac{i \sqrt{2}}{2}\left[(-1)^{q} A_{+q}^{(k)}-A_{-q}^{(k)}\right]=(-1)^{q} \sqrt{2} \operatorname{Im}\left(A_{+q}^{(k)}\right), \quad q>0, \\
& A_{0}^{\{k\}}=A_{0}^{(k)} .
\end{aligned}
$$

In Section 3 we will discuss the most common methods of preparation of polarized distributions of $j$ and $r$, but we consider first the description of the intrinsic polarization.

\subsection{Intrinsic polarization distributions.}

As we have seen, the intrinsic polarization distribution contains all of the dynamical information about the dependence of the reaction integral or differential cross section on the anisotropies of the rotational angular momentum and molecular axis distributions of the reactants. 
Quasi-classical trajectory (QCT) and QM dynamical calculations are the main theoretical methods used to determine polarization moments. The former can be considered as an approximation, sometimes inaccurate, to the latter. In addition, the effect of the electronic orbital or spin polarization cannot be tackled within the QCT approach. A crucial point, however, is to establish a common mathematical language whereby QM and QCT results can be directly compared. As might be expected, that language is that of the polarization moments.

In contrast to the laboratory (LAB) reference frame normally used to describe the preparation of the external polarization of angular momenta, the intrinsic polarization is usually described in the collision frame, for which the $z$-axis is along the initial relative velocity, $\boldsymbol{k}$, and the $x z$ reference plane contains the final relative velocity, $\boldsymbol{k}^{\prime}$. This is equivalent to the centre-of-mass (CM) frame usually employed in crossed molecular beam experiments. ${ }^{1}$

Similarly to the description of the extrinsic polarization, the dynamical, intrinsic information can be cast in terms of a normalized PDF, which in turn can be expressed as a multipole expansion. However, in contrast to the extrinsic preparation of a given (asymptotic) reactant polarization, the intrinsic description goes one step further and is defined only as long as the reaction takes place. ${ }^{9}$ It tells us under what circumstances (reactant polarizations) the reaction products are formed in specific final states and/or scattered into a given scattering angle. Equivalently, it determines the best reactant polarization to be used to maximize the amount of products born in a specific quantum state and scattering angle.

In the QCT description, the PDF would represent the probability of the reaction products being scattered into a given infinitesimal interval $[\theta, \theta+\mathrm{d} \theta]$ when the direction of the rotational angular momentum is specified by $\theta_{j}$ and $\phi_{j}$. This PDF may refer to a specific final quantum state of the products or to the overall reaction. In terms of the polarization moments this PDF can be written as ${ }^{9}$

$$
P\left(\theta, \theta_{j}, \varphi_{j}\right)=\sum_{k=0}^{k_{\max }} \sum_{q=-k}^{k} \frac{2 k+1}{4 \pi} S_{q}^{(k)}(\theta) C_{k q}^{*}\left(\theta_{j}, \varphi_{j}\right)
$$

The $\theta$ dependent polarization moments, $S_{q}^{(k)}(\theta)$, are called polarization dependent differential cross sections (PDDCSs). In particular, the $k=0, q=0$ moment (the monopole) represents the normalized differential cross section (DCS), i.e. the angular scattering distribution in the CM collision frame:

$$
S_{0}^{(0)}(\theta)=P(\theta)=\frac{2 \pi}{\sigma} \frac{\mathrm{d} \sigma}{\mathrm{d} \omega},
$$


where $\sigma$ is the integral cross section for unpolarized reactants. Note the similarities, but also the important differences, between the extrinsic and intrinsic polarization moments. In the former case, the $A_{0}^{(0)}$ moment was simply a normalization constant. In the intrinsic case, the monopole moment is the DCS, which contains a great deal of information about the dynamics of the scattering process for unpolarized reactants. Note that the integral of Eq. (5) over scattering angle yields the classical PDF $P\left(\theta_{j}, \varphi_{j}\right)$, describing the integral steric effect, which is readily expressed in terms of scattering angle integrated polarization moments, $s_{q}^{(k)}$. $^{.}$

Another important quantity is the conditional reaction $\mathrm{PDF},{ }^{15,16}$

$$
P\left(\theta_{j}, \varphi_{j} \mid \theta\right)=\frac{P\left(\theta, \theta_{j}, \varphi_{j}\right)}{P(\theta)}=\sum_{k=0}^{k_{\max }} \sum_{q=-k}^{k} \frac{2 k+1}{4 \pi} \frac{S_{q}^{(k)}(\theta)}{S_{0}^{(0)}(\theta)} C_{k q}^{*}\left(\theta_{j}, \varphi_{j}\right),
$$

which quantifies the reaction probability, under the condition that products are scattered at angle $\theta$, that the reactant rotational angular momentum, $\boldsymbol{j}$, will lie along the direction $\theta_{j}, \varphi_{j}$. The coefficients $S_{q}^{(k)}(\theta) / S_{0}^{(0)}(\theta)$ are the renormalized PDDCSs.

As in the case of the intrinsic PDFs, the various PDFs of Eqs. (5) and (7) suffer from the same limitations. Using the scattering angle resolved PDF, $P\left(\theta, \theta_{j}, \varphi_{j}\right)$, as an example, the correct QM expression is ${ }^{12,13}$

$$
P\left(\theta, \theta_{j}, \varphi_{j}\right)=\sum_{k=0}^{k_{\max }} \sum_{q=-k}^{k} \frac{2 k+1}{4 \pi} S_{q}^{(k)}(\theta)\langle j j, k 0 \mid j j\rangle C_{k q}^{*}\left(\theta_{j}, \varphi_{j}\right)
$$

It can be shown that when the intrinsic PDF of the rotational angular momentum is given by Eq. (8), the corresponding distribution of internuclear axis is given by ${ }^{12,13}$

$$
P\left(\theta, \theta_{r}, \varphi_{r}\right)=\sum_{k=0}^{k_{\max }} \sum_{q=-k}^{k} \frac{2 k+1}{4 \pi} S_{q}^{(k)}(\theta)\langle j 0, k 0 \mid j 0\rangle C_{k q}^{*}\left(\theta_{r}, \varphi_{r}\right)
$$

Eq. (9) represents the intrinsic reaction probability into a scattering angle $\theta$ for a given internuclear axis direction, $\theta_{r}, \varphi_{r}$, when the corresponding reaction probability as a function of the angular momentum is given in terms of the $j$-PDDCSs, $S_{q}^{k}(\theta)$, according to Eq. (8). According to this expression, since the $\langle j 0, k 0 \mid j 0\rangle \mathrm{CG}$ coefficients are zero unless $k$ is even, the intrinsic $r$-distribution cannot display any orientation if the reactant is in a well-defined initial $j$ state, and in the absence of an external field.

It is possible to use QCT or QM methods to determine the $S_{q}^{(k)}(\theta)$ PDDCSs that define the intrinsic polarization for the scattering process. The details of such calculations are beyond the scope of this 
Perspective article, but an accessible review of such methods can be found in Ref. [10]. Suffice to say that the QM PDDCSs and polarization moments can be calculated directly from the scattering matrix. The QCT PDDCSs and polarization moments can likewise be obtained readily from expectation values of the appropriate multipoles over ensembles of classical trajectories.

\subsection{How the experimental preparation affects the measurable cross section.}

The next obvious step is to combine the extrinsic and intrinsic polarizations. From the theoretical point of view, this means predicting the measured integral or differential cross section subject to a given polarization of the reactants. This is equivalent, although in the reverse direction (from the experimental point of view) to deriving the intrinsic (and hence dynamical) polarization from a series of measurements with different preparations of the angular momentum or internuclear axis distributions.

The first thing to note is that in general the frames that have been used in the extrinsic and intrinsic description do not coincide. Although simulations can be (and sometimes have to be) carried out in the LAB system, it is preferable to determine the DCS in the collision frame for a specific polarization.

Suppose that the direction of the light polarization or electric field vectors is defined in the collision frame by two angles $\theta_{E}$ and $\phi_{E}$. If the polarization parameters that define the polarization of the angular momentum in the LAB frame, with the $Z$-axis along $\boldsymbol{E}$, are $A_{q}^{(k)}$, we simply need a rotation to obtain them in the collision frame:

$$
a_{q}^{(k)}=\sum_{q^{\prime}} D_{q q^{\prime}}^{(k) *}\left(\phi_{E}, \theta_{E}, 0\right) A_{q^{\prime}}^{(k)}
$$

It is easy to show that the observable DCS resulting from the set of intrinsic polarization moments, $a_{q}^{(k)}$, will be ${ }^{9}$

$$
\left[\frac{\mathrm{d} \sigma}{\mathrm{d} \omega}\right]_{\mathrm{pol}}=\frac{\sigma}{2 \pi} \sum_{k} \sum_{q}(2 k+1) a_{q}^{(k)}\left[S_{q}^{(k)}(\theta)\right]^{*},
$$

where the sub-index 'pol' indicates that the reactants are polarized. When there is no polarization, that is when $a_{q}^{(k)}=\delta_{k, 0} \delta_{q, 0}$, the usual unpolarized DCS is recovered, as given by Eq. (6).

In many cases, due to cylindrical symmetry of $\boldsymbol{j}$ about the field $\boldsymbol{E}$ only the $A_{0}^{(k)}$ terms are non-zero, and then

$$
a_{q}^{(k)}=C_{k q}\left(\theta_{E}, \phi_{E}\right) A_{0}^{(k)}
$$


In this case, Eq. (11) becomes

$$
\left[\frac{\mathrm{d} \sigma}{\mathrm{d} \omega}\right]_{\phi_{E}}^{\theta_{E}}=\frac{\sigma}{2 \pi} \sum_{k} \sum_{q}(2 k+1)\left[S_{q}^{(k)}(\theta)\right]^{*} C_{k q}\left(\theta_{E}, \phi_{E}\right) A_{0}^{(k)}
$$

where the angles that define the direction of the electric field, $\theta_{E}, \phi_{E}$, for example, serve to define the specific polarization ${ }^{17}$ of the DCS.

Similarly, one can define a polarized integral cross section that will only be dependent of the polar angle, $\theta_{E}$

$$
[\sigma]^{\theta_{E}}=\sigma \sum_{k}(2 k+1) s_{0}^{(k)} P_{k}\left(\cos \theta_{E}\right) A_{0}^{(k)}
$$

where $P_{k}(\cos \theta) \equiv C_{k 0}(\theta, 0)$ is the Legendre polynomial of degree $k$, and $s_{0}^{(k)}$ represents the integral of the $S_{q}^{(k)}(\theta)$ PDDCSs over scattering angle. Notice that only the polarization parameters with $q=0$ appear in this equation as a result of integrating over $\phi_{E}$ in Eq. (13).

Many of the examples described in the following sections will be concerned with the orientation and alignment of open-shell diatomic molecules. While much can be learned from the general treatment outlined above, the electric fields used to select and orient such molecules mix the initial quantum states of the system, and so the clear division between intrinsic and extrinsic moments becomes less transparent and more complicated. We defer discuss of orientation and alignment of open-shell diatomic molecules until Section 3.3.

Now furnished with the theoretical tools described above, we can consider the most common ways to polarize reactant molecules.

\section{Experimental aspects}

Here we will concentrate primarily on preparing reactant molecules, focusing on techniques that allow either $\boldsymbol{r}$ or $\boldsymbol{j}$ to be polarized. ${ }^{4,10,18}$ By defining the initial polarization of either the bond axis $(\boldsymbol{r})$ or the angular momentum vector $(j)$, it becomes possible to measure integral and differential steric effects which quantify two and three vector correlations, respectively. 


\subsection{Optical polarization}

The absorption of light is an inherently anisotropic process, which leads to the polarization of both excited and ground state molecules. ${ }^{19-21}$ For a closed shell diatomic molecule in a ${ }^{1} \Sigma$ electronic state and a specific $|j m\rangle$ rotational state, the probability of the bond axis, $r$, pointing along a particular direction specified by angles $\Theta_{r}, \Phi_{r}$ is given by ${ }^{6}$

$$
P_{j m}\left(\Theta_{r}, \Phi_{r}\right)=\left|Y_{j m}\left(\Theta_{r}, \Phi_{r}\right)\right|^{2} .
$$

The probability that the diatomic will exist in state $|j m\rangle$ after absorption of a polarized photon is given by $\left|\left\langle j^{\prime \prime} m^{\prime \prime}, 10 \mid j m\right\rangle\right|^{2}$. Assuming that the diatomic reactant is initially unpolarized, the probability of finding $\boldsymbol{r}$ along a particular direction is a product of these two terms, ${ }^{6,22}$

$$
P\left(\Theta_{r}, \Phi_{r}\right)=\frac{2 j+1}{4 \pi\left(2 j^{\prime \prime}+1\right)} \sum_{m=-j^{\prime \prime}}^{j^{\prime \prime}}\left\langle j^{\prime \prime} m^{\prime \prime}, 10 \mid j m\right\rangle^{2}\left|C_{j m}\left(\Theta_{r}, \Phi_{r}\right)\right|^{2}
$$

which, after introduction of the molecular axes alignment parameter $\mathscr{A}_{2}(j)$, can be rewritten as ${ }^{6}$

$$
P\left(\Theta_{r}, \Phi_{r}\right)=\frac{1}{4 \pi}\left[1+\mathscr{A}_{2}(j) P_{2}\left(\cos \Theta_{r}\right)\right]
$$

The $\mathscr{A}_{2}(j)$ parameter is related to the rotational polarization moment by $\mathscr{A}_{2}(j)=5\langle j 020 \mid j 0\rangle a_{0}^{(2)}$. By using linearly polarized light, we can therefore only align a molecule, not orient it. In the case of circularly polarized light it can be shown that while $j$ can be oriented, the bond axis $r$ can still only be aligned.

For a symmetric top molecule, the polarization along the molecular axes is given by $6,10,22$

$$
\begin{aligned}
P\left(\Theta_{r}, \Phi_{r}\right) & =N \sum_{m^{\prime \prime}=-j^{\prime \prime}}^{j^{\prime \prime}} \sum_{m=-j}^{j}\left\langle j^{\prime \prime} m^{\prime \prime}, 1 Q \mid j m\right\rangle^{2}\left\langle j^{\prime \prime} k^{\prime \prime}, 1 \Delta k \mid j k\right\rangle^{2} \\
& \times\left[\left|D_{m k}^{j}\left(\Theta_{r}, \Phi_{r}, \chi_{r}\right)\right|^{2}+\left|D_{m-k}^{j}\left(\Theta_{r}, \Phi_{r}, \chi_{r}\right)\right|^{2}\right]
\end{aligned}
$$

with $N$ a normalization constant, and $Q$ a light polarization index, which is 0 for linearly polarized light and \pm 1 for circularly polarized light, and where $\Delta k=0$ for parallel transitions and \pm 1 for perpendicular transitions. As in the diatomic case, in the absence of an external field the symmetry axes can be aligned but not oriented, either with circularly or linearly polarized light. 
Following early work from Zare and coworkers, ${ }^{23}$ Wang et al. ${ }^{24-26}$ have recently used optical preparation with an infrared laser to pre-align $\mathrm{CHD}_{3}$ molecules to unravel the steric preferences of the reaction of $\mathrm{CHD}_{3}$ with $\mathrm{Cl}$. Fig. 1 shows the setup of their experiment, which made use of optical alignment to control the initial angular momentum vector of the reacting $\mathrm{CHD}_{3}$ (see Section 4.2).

The bond axis can also be polarized by absorption of two or more photons. ${ }^{27,28}$ Stimulated Raman Pumping has been used by Zare and coworkers ${ }^{29}$ to promote HD to well-defined $|j m\rangle$ states. These states were shown to have a well-defined $j$ alignment either along or perpendicular to the electric vector of the pump laser. Recently, Mukherjee and Zare have developed the Stark-induced Adiabatic Raman Passage (SARP) method for preparing polarized samples. ${ }^{30-33}$ The SARP method has been shown to allow almost complete population transfer from the ground state of $\mathrm{H}_{2}$ to an excited rovibrational state with a well defined $m=0$ polarization. Population transfer to $|v j m\rangle=|v 2 \pm 2\rangle$ was also shown to be achievable. A similar method has also been demonstrated on heteronuclear diatomics using the related stimulated Raman adiabatic passage $\left(\mathrm{STIRAP}^{34}\right)$ technique, ${ }^{30,32}$ which allows control of the orientation and alignment of excited states of heteronuclear diatomic molecules.

A different way of using photons to align a molecular ensemble is to use an intense, polarized laser field to generate an strong, localized electric field, and thereby to exploit the molecular Stark effect. The control of molecular alignment ${ }^{35-38}$ and orientation ${ }^{39-41}$ through intense laser pulses has received much attention recently in the literature, and has been the subject of several reviews. ${ }^{42,43}$ These methods, though extremely powerful and general, have yet to be applied to crossed molecular beam scattering experiments.

\subsection{Brute force orientation}

It has long been known that a molecule can be oriented in an electric field by virtue of the interaction with its dipole moment. However, the rotation of the molecule almost averages out the orientation of the molecule unless strong fields are employed. ${ }^{44-48}$ With the advent of cold molecular beam sources, very low rotational temperatures became accessible, and brute force orientation of molecules in static electric fields then became possible with electric field strengths in the range 10 to $20 \mathrm{kV} \mathrm{cm}^{-1}$, depending on the magnitude of the molecular dipole moment. ${ }^{44-50}$

When a polar molecule is placed in an electric field, the bond axis distribution becomes spatially oriented. ${ }^{10,50-54}$ Using this method, the molecule can be both aligned and oriented. ${ }^{5}$ Upon entering an 
external field, the molecule's rotational eigenstates typically adiabatically follow the electric field it is exposed to. A linear combination of rotational states is created in the perturbing field, as the molecular dipole attempts to orient with the field against the rotational motion. Depending on the field strength, the molecule can be thought of as residing in a 'pendular state', ${ }^{46-48,55}$ which effectively reduces the molecular orientation achievable in the applied field. Thus the orientation achievable is dependent on the initial rotational temperature of the molecule, $T_{\text {rot }},{ }^{44,46,48}$ with lower rotational temperatures favouring better molecular orientation. It has been shown ${ }^{44-48}$ that the angular distribution of the bond axis is well-approximated by the equation

$$
P\left(\cos \Theta_{r}\right)=\frac{1}{2}\left[1+\mathscr{A}_{1} \cos \Theta_{r}\right]
$$

where $\mathscr{A}_{1}=a E / T_{\text {rot }}$, with $a$ a system specific constant, and $E$ the field strength. The brute force method is therefore most efficient at orienting $\boldsymbol{r}$ when the initial rotational temperature is low, and diminishes as the initial molecular rotational temperature increases.

The brute force orientation and alignment method has been widely used in scattering experiments, as will be illustrated in many of the case studies described in Section 4 .

\subsection{Orientation and alignment coupled with hexapole state selection}

\subsubsection{General background}

The basis of hexapole state-selection and molecular orientation is the first order Stark effect, described in detail in previous work. ${ }^{53,56-59}$ For the selection of symmetric top molecules in state $|j m k\rangle,{ }^{60}$ the first order perturbation correction to the molecular energy in the inhomogeneous hexapole electric field is given by

$$
W^{(1)}=-\mu_{e} E\left\langle j m k\left|\cos \Theta_{r}\right| j m k\right\rangle=-\mu_{e} E \frac{m k}{j(j+1)} .
$$

If $k=0$, as is always the case for closed-shell diatomic molecules, the first order Stark effect disappears. Higher order effects can in principle be used to focus such molecules, but due to the second order nature of these effects, the focussing force even at high electric field strengths is very small. ${ }^{52,61}$

As can be seen from Fig. 2, the hexapole consists of an arrangement of six rods, each with alternating 
electric charge. The force felt by a molecule in the hexapole is given by ${ }^{62}$

$$
F_{\text {hex }}=\mu_{e}\left\langle\cos \Theta_{r}\right\rangle \frac{6 V_{0} R}{R_{0}^{3}}
$$

The hexapole (and also a quadrupole in the case of a positive quadratic Stark effect ${ }^{52}$ ) therefore behaves like a lens, focusing molecules with $\left\langle\cos \Theta_{r}\right\rangle<1$ into a focal point, thereby increasing the number density of these state-selected molecules in the interaction region by up to two orders of magnitude. ${ }^{52,53,56}$ The exact magnification factor is determined by the hexapole rod voltage, the geometry of the hexapole, and the effective size of the molecular beam source. ${ }^{63}$

By means of a set of orientation rods, as illustrated in Fig. 3, which generate a uniform static electric guiding field, the state-selected molecules emerging from the hexapole state-selector can typically be oriented in space virtually adiabatically. ${ }^{52,53,56,64}$

\subsubsection{Orientation and scattering of $\mathrm{NO}(\mathrm{X})$ and $\mathrm{OH}(\mathrm{X})$}

The ground state electron configuration of the nitric oxide (NO) molecule is given by

$$
1 \sigma^{2} 1 \sigma^{* 2} 2 \sigma^{2} 2 \sigma^{* 2} 1 \pi^{4} 3 \sigma^{2} 1 \pi^{* 1}
$$

The unpaired electron in the $1 \pi^{*}$ orbital carries a single unit of electronic orbital angular momentum, $\boldsymbol{L}=1$, which makes a projection onto the molecular axes of $\Lambda= \pm 1 .{ }^{65}$ The electron spin in the ${ }^{2} \Pi$ state is $S=\frac{1}{2}$, with a projection onto the molecular axes $\Sigma= \pm 1 / 2$. For $\mathrm{NO}(\mathrm{X})$, the coupling between the molecular framework and electron angular momenta is best described by Hund's case (a) coupling. $\boldsymbol{R}$ couples to $\Omega=\Lambda+\Sigma$, with the resultant vector being the total angular momentum apart from nuclear spin, $j . \Omega$ can take values $\frac{1}{2}$ or $\frac{3}{2}$, labelled as $F_{1}$ and $F_{2}$, respectively, giving rise to the lower and upper spin-orbit manifolds.

For the hydroxyl radical, $\mathrm{OH}$, the ground state electronic configuration is given by

$$
1 \sigma^{2} 1 \sigma^{* 2} 2 \sigma^{2} 1 \pi^{3}
$$

$\mathrm{OH}(\mathrm{X})$ also has a ${ }^{2} \Pi$ ground state, but differs from $\mathrm{NO}(\mathrm{X})$ in that the $j=\Omega=\frac{3}{2}$ state is lower in energy than the $j=\Omega=\frac{1}{2}$ state. 
The wavefunctions for the $|j m \Omega\rangle$ states are related to the Wigner rotation matrices, and can be written as 66

$$
|j m \Omega\rangle=\sqrt{\frac{2 j+1}{4 \pi}} D_{m \Omega}^{j *}(\Phi, \gamma, 0),
$$

with $m$ the projection of $j$ onto the quantization axis. The states defined in Eq. (24) are not eigenfunctions of the system Hamiltonian. The correct description of the pure Hund's case (a) wavefunction for the ground state of $\mathrm{NO}$ and $\mathrm{OH}$ is given by ${ }^{6}$

$$
|j m \Omega \varepsilon\rangle=\frac{1}{\sqrt{2}}[|j m+\bar{\Omega}\rangle+\varepsilon|j m-\bar{\Omega}\rangle] .
$$

$\bar{\Omega}$ is the absolute value of $\Omega$, and $\varepsilon= \pm 1$ is a parity index, related to the parity by $p=(-1)^{j-\varepsilon / 2}$. The two states of differing parity are referred to as a $\Lambda$-doublet, and are very closely spaced in energy, meaning that even in the coldest of molecular beam expansions the rotational ground state has nearly equal populations of each parity level. The two levels are often designated by their spectroscopic labels $e$ and $f$, which refer to the lower and upper $\Lambda$-doublet levels, respectively.

The interaction of the $\mathrm{OH}(\mathrm{X})$ or $\mathrm{NO}(\mathrm{X})$ with an incoming atom is described by motion on two potential energy surfaces (PESs). The approach of the rare gas to the radical reduces the symmetry from $C_{\infty \nu}$ to $C_{s}$, and the triatomic electronic states are denoted $A^{\prime}$ and $A^{\prime \prime}$ according to their reflection symmetry with respect to the triatomic plane. It is often more convenient to rewrite the two potentials as half-sum, $V_{\text {sum }}$, and half-difference, $V_{\text {diff }}$, potentials, involving linear combinations of the $A^{\prime}$ and $A^{\prime \prime}$ states. ${ }^{66-69}$ For Hund's case (a) coupling, spin-orbit conserving collisions have been shown to take place on the $V_{\text {sum }}$ potential, whilst spin-orbit changing collision are coupled by $V_{\text {diff. }}{ }^{66}$

Because open-shell diatomic molecules behave as symmetric tops, hexapole state-selection and orientation methods can also be used to orient molecules such as $\mathrm{OH}(\mathrm{X})$ or $\mathrm{NO}(\mathrm{X}) .{ }^{70}$ Hexapole state-selection of the molecules is necessary before they enter a region with a uniform orienting electric field. In $\mathrm{NO}(\mathrm{X})$, the $j=1 / 2, \bar{\Omega}=1 / 2, \varepsilon=-1(f)$ level is selected in the hexapole state-selector. This quantum state is a low field seeking state, such that the N-end of the NO points towards the negative electrode. ${ }^{57,58}$ The other $\Lambda$-doublet level $(j=1 / 2, \bar{\Omega}=1 / 2, \varepsilon=1(e))$ would be oriented in the other direction in the static electric field. Therefore, orientation of $\mathrm{NO}(\mathrm{X})$, or other open-shell molecules, is only possible by ensuring state purity at the point of molecular orientation. 
Recent experiments on state-selected NO employed the four-rod assembly shown in Fig. 3 to generate the static orienting electric field. Assuming the DC electric field lies along the relative velocity, $k$, the eigenstate wavefunction of $\mathrm{NO}(\mathrm{X})$ in the field is given by a linear combination of the field free $\varepsilon=-1$ and $\varepsilon=+1 \Lambda$-doublet states ${ }^{57,58,71-73}$

$$
|j m \Omega E\rangle=\frac{1}{\sqrt{2}}[\alpha|j m \Omega \varepsilon=1\rangle+\beta|j m \Omega \varepsilon=-1\rangle] .
$$

The real and positive valued mixing coefficients $\alpha$ and $\beta$ are given in terms of the electric field strengths, where in this case the coefficient $\alpha$ goes to zero as the field goes to zero. ${ }^{57,58,71-73}$ As mentioned in Section 2.3, note further that in the open-shell case the separation into intrinsic and extrinsic moments, introduced in Section 2, is complicated by the fact that the static electric field causes a mixing of $\Lambda$ doublet levels.

For NO state-selected in the $j=1 / 2$ level, the normalized probability distribution of the cosine of the angle between the dipole moment of $\mathrm{NO}(\mathrm{X})$ and the electric field $\left(\Theta_{\mu E} \equiv \Theta_{r}\right)$ is given by a simple equation in $\alpha$ and $\beta$

$$
P\left(\Theta_{\mu E}\right)=\frac{1}{2}\left[1+\alpha \beta \cos \Theta_{\mu E}\right] .
$$

Once the orientation specified by Eq. (27) has occurred, scattering with the partner atom takes place and a detection scheme is used to infer the collision outcome. The polarity of the rods can be switched so the $\mathrm{N}$ and $\mathrm{O}$ end collisions can be measured in an alternating cycle to minimize the effects of experimental instability. With moderate field strengths, a relatively high degree of orientation of the $\mathrm{NO}(\mathrm{X})$ bond axis is possible. As we will see, similar experimental methods can also be used to study orientation effects in reactions involving other open-shell diatomic molecules, such as $\mathrm{OH}(\mathrm{X}) .{ }^{57}$ As discussed in Section 4.2, using these oriented reactant molecules, combined with fast switching electronics, allows the sterically resolved $\boldsymbol{k}-\boldsymbol{r}-\boldsymbol{k}^{\prime}$ vector correlation, corresponding to the differential steric asymmetry, to be probed. Appropriate integration of the orientated or aligned DCSs allows recovery of the integral steric asymmetry, discussed in Section 4.1. 


\subsection{Other experimental considerations}

Coupling the above methods for preparing polarized molecular ensembles with crossed molecular beam experiments allows the stereodynamics of inelastic and reactive scattering encounters to be probed quantitatively.

In the earliest crossed molecular beam experiments with polarized reactants, detection with moveable time-of-flight detectors was used to record differential cross sections at discrete angles, and to obtain the integral steric asymmetry ${ }^{70}$ (see Section 4.1). ter Meulen ${ }^{72,74,75}$ and Stolte ${ }^{57,58,76}$ and their coworkers have subsequently used laser-induced fluorescence (LIF) to great effect to measure the integral steric asymmetries for several inelastic scattering systems (see Section 4.1). Although LIF has been used to measure DCSs, it is not that easy to employ in scattering angle-resolved measurements, and so differential steric effects have not been measured using this detection method.

In the most modern crossed molecular beam machines, velocity-map ${ }^{77}$ ion imaging ${ }^{78}$ has been combined with advanced quantum state selection techniques to obtain complete angular scattering distributions (see for example Refs. [79-90]). Velocity-map ion imaging provides a very effective technique for obtaining the differential and integral steric asymmetries, when coupled with hexapole state selection and guiding rod orientation. By coupling these advanced methods with the state preparation described above, it is possible to measure sterically resolved angular distributions and integral steric asymmetries with more precision than ever before.

\section{Case studies}

\subsection{Integral steric asymmetry}

The influence that molecular orientation has on the outcome of a collision can be quantified by a dimensionless integral steric asymmetry parameter, $S$. For the collision of a diatomic molecule, AB, with an atom or molecule the integral steric asymmetry is defined as

$$
S=\frac{\sigma_{\mathrm{A}}-\sigma_{\mathrm{B}}}{\sigma_{\mathrm{A}}+\sigma_{\mathrm{B}}} \times 100
$$


where $\sigma_{\mathrm{A}}$ and $\sigma_{\mathrm{B}}$ are, for example, the integral cross sections for the oriented molecule. A similar expression holds for reactions involving side-on versus end-on molecular alignment of reactants. In the case of oriented reactants, a positive value of $S$ indicates a preference for 'A-end' collisions and a negative value indicates that inelastic scattering or reaction is favoured following collision at the 'B-end' of the molecule. It should be mentioned that it is also possible to measure the integral steric asymmetry for the collision of a molecule with a variety of solid surfaces. ${ }^{91,92}$ However, the effect of molecular orientation on such collisions is beyond the scope of the current Perspective article, and will not discussed further here.

Early work on the influence of steric effects on the asymmetry of chemical reactivity, performed by Bernstein and coworkers, focused on the 'reactive asymmetry' of the reaction between $\mathrm{CH}_{3} \mathrm{I}$ and $\mathrm{Rb}$. A steric cone of non-reaction was observed in which the methyl group blocks the approach of the Rb atom. ${ }^{93,94}$ The reactive asymmetry was observed to be large for backscattered products (RbI), whilst forward scattering was less orientation-dependent. Loesch and coworkers also reported the integral steric asymmetry for the reaction of $\operatorname{Sr}+\operatorname{HF}(v=1, j=1) \rightarrow \operatorname{SrF}\left(v^{\prime}, j^{\prime}\right)+$ H performed with molecular reactant alignment favouring either side-on or end-on attack on the HF molecule. It was found that there was a small but significant alignment effect for collisions populating different final vibrational states. ${ }^{95}$ In a subsequent study, the side-on favouring reaction geometry at a collision energy $E_{\mathrm{col}}=230 \mathrm{meV}$ was observed to gradually change into an end-on favouring one at $E_{\mathrm{col}}=625 \mathrm{meV} .{ }^{96}$ Further examples of steric asymmetries for chemical reactions are given in Section 4.2.

One of the most common measurements of the integral steric asymmetry has been for inelastic scattering. Inelastic collisions of $\mathrm{NO}\left(X^{2} \Pi\right)$ with $\mathrm{Ar}$ are often used as a prototype for studying the collision of open shell molecules with closed shell atoms, and many measurements of the integral steric asymmetry have focused on this system. Detailed experimental and theoretical studies have been carried out by Stolte $^{57,71,76,79}$ and Brouard ${ }^{73}$ and their groups, in which the $\mathrm{NO}(\mathrm{X})$ molecules were state selected using a hexapole in the $\Omega=0.5, j=0.5, f$ state, prior to being oriented in the interaction region using a static electric field, as discussed in Section 3. Typical values of the average bond axis orientation achieved in these experiments correspond to $\left\langle\cos \Theta_{r}\right\rangle \sim 0.3$.

Fig. 4 shows a comparison between QM and QCT theoretical and experimental integral steric asymmetry for spin-orbit conserving $\left(j=0.5, \Omega=0.5 \rightarrow j^{\prime}, \Omega^{\prime}=0.5\right)$ transitions into the $e \Lambda$-doublet state. ${ }^{73}$ Good agreement between the experimental values and QM calculations is observed for all values of 
$\Delta j=j^{\prime}-j$. The experimental data show marked oscillations between positive ( $\mathrm{N}$-end preference) and negative (O-end preference) steric asymmetries for $\Delta j=$ odd and $\Delta j=$ even, respectively, particularly for transitions with $5 \leq \Delta j \leq 12$. Since QCT calculations cannot reproduce the experimental alternation in $\Delta j$, whilst the QM calculations can, the oscillations have been interpreted in terms of a $\mathrm{QM}$ interference effect. ${ }^{71,73}$ Further insight was obtained using a quasi-quantum treatment, from which it is found that the alternation in sign is due to quantum interference from 'trajectories' scattering off the two ends of the NO molecule. ${ }^{79}$ For high rotational excitation $(\Delta j \gtrsim 12), S$ becomes large and positive, indicating that $\mathrm{N}$-end collisions preferentially populate high rotational states. This is reproduced by both the QM and QCT calculations, and can be rationalized in terms of a classical ball-and-stick model: since the Pauli repulsion induced by the unpaired electron charge density in the $1 \pi^{*}$ orbital favours the $\mathrm{N}$-end over the O-end ${ }^{97}$ and the centre-of-mass of the NO molecule is displaced slightly towards the oxygen, a larger torque may be applied by collision at the $\mathrm{N}$-end of the molecule, leading to somewhat greater rotational excitation. ${ }^{73}$ The increase in the magnitude of $S$ with increasing $\Delta j$ for spin-orbit conserving collisions may be understood by considering the near-homonuclear nature of the NO molecule; as the potential becomes more homonuclear, scattering becomes less dependent on the reactant orientation. For transitions with low $\Delta j$, which are governed by the coefficient of the second Legendre moment in the potential expansion, $V_{20}$, the interaction potential is near-homonuclear, leading to small steric asymmetries. As $\Delta j$ increases collisions sample the more anisotropic regions of the PES, leading to larger observed values of $S .{ }^{68,71}$

Experiments to understand the effect of the underlying potential on the integral steric asymmetry were performed by comparing inelastic collisions of state-selected $\mathrm{NO}(\mathrm{X})$ with $\mathrm{Ar}$ and He. ${ }^{58,98}$ Whilst the alternation in the sign of the integral steric asymmetry is observed in both systems, the magnitude of $S$ was found to be considerably larger (as well as more regular in structure) for NO-He scattering than for NO-Ar. This, along with calculations on modified test potentials, ${ }^{71}$ suggests that the repulsive part of the underlying PES has a stronger effect on $S$, since the NO-He PESs are less attractive than for NO-Ar.

Work by the group of ter Meulen has focused on the integral steric asymmetry of the $\mathrm{OH}(\mathrm{X})$ radical prepared in the $|v=0, \Omega=1.5, j=1.5\rangle$ state. In the study of collisions of $\mathrm{OH}$ with $\mathrm{Ar}$, the overall $\mathrm{OH}$ orientation was found to be $\left\langle\cos \Theta_{r}\right\rangle \sim 0.46$ and $\left\langle\cos \Theta_{r}\right\rangle \sim 0.55$ for single and double hexapole state selection, respectively. ${ }^{72,74,75}$ The difference in the measured steric asymmetry between the single and double hexapole can be seen in Fig. 5: for transitions to all but the $\left|\Omega^{\prime}=1.5, f\right\rangle$ state (upper right panel), a larger absolute value is observed from the double hexapole. Due to higher purity of initial state selection 
and a higher degree of orientation achieved in the static field, it is most useful to consider the double hexapole selection steric asymmetry. Low $\Delta j$ excitation shows a weak preference for O-end collisions (positive $S$ ) whilst excitation to high $j^{\prime}$ states has a strong preference for H-end collisions (negative $S$ ). The steric asymmetry becomes increasingly negative with increasing $\Delta j$, favouring $\mathrm{H}$-end collisions, which can be understood in terms of a similar classical ball-and-stick model to that described for NO + Ar collisions. In the $\mathrm{OH}+\mathrm{Ar}$ system, the steric asymmetry depends on the final $\Lambda$-doublet state under consideration: for spin-orbit conserving transitions to $e$ states, $S$ is more negative compared to transitions to $f$ states (compare the upper left and upper right panels of Fig. 5), a trend which is is reversed for spinorbit changing transitions (see the lower panels of Fig. 5). Although it was found that the experimental results agreed reasonably well with QM calculations, currently there is no simple explanation for this behaviour.

There are some marked differences between the integral steric asymmetries observed for collisions of $\mathrm{NO}^{57,73,76}$ and $\mathrm{OH}$ with Ar. ${ }^{72,74,75}$ Whilst there is a significant difference in $S$ for $e$ and $f$ final states for $\mathrm{OH}$, this is only minor for NO. This contrasting behaviour is thought to be due to differences in the rotational level coupling according to Hund's cases, and the fact that NO is a near homonuclear diatomic molecule. ${ }^{57,58,71,72,76}$ Furthermore, the oscillation in the sign of $S$ with $\Delta j$ observed for collisions with $\mathrm{NO}+\mathrm{Ar}$ is not present for $\mathrm{OH}+\mathrm{Ar}$. As we have seen, the oscillations in the case of $\mathrm{NO}+\mathrm{Ar}$ arise from the near homonuclear nature of the PESs describing that system, which leads to an interference between scattering off either end of the molecule. ${ }^{79}$ This interference is therefore not seen in the much more highly heteronuclear $\mathrm{OH}+\mathrm{Ar}$ system. The increased magnitude of $S$ observed for OH compared to NO is likely partly due to the higher level of orientation, along with the larger charge and mass asymmetry present for the $\mathrm{OH}$ radical compared with $\mathrm{NO}$.

More recent work has focused on the determination of the integral steric asymmetry for diatom-diatom scattering. ter Meulen's group has investigated the integral steric asymmetry of the inelastic scattering of $\mathrm{OH}(v=0, j=1.5, f)$ by $\mathrm{HX}(v=0, j<4)$, where $\mathrm{X}=\mathrm{I}^{99}, \mathrm{Cl}^{100}$ and $\mathrm{Br},{ }^{101}$ as shown in Fig. 6. For all scattering partners, $S$ depends on the spin-orbit manifold and the $\Lambda$-doublet level of the final state. It is interesting to note, that unlike in other systems (for example $\mathrm{OH}+\mathrm{X}, \mathrm{X}=\mathrm{Ar}, \mathrm{N}_{2}$ and $\mathrm{CO},{ }^{72,75}$ ) the value of $S$ for $\mathrm{OH}+\mathrm{HX}$ does not vary monotonically with $\Delta j$. This cannot be understood using a simple classical ball-and-stick model, and is thought to reflect the balance between intermolecular dispersion interactions and the dipolar and quadrupolar moments present in the HX systems. Considering spin-orbit conserving 
transitions, to final $e \Lambda$ doublet states, as shown in the top right panel of Fig. 6, a weak preference for O-end collisions (positive $S$ ) is observed for all collision partners, whilst for $f$ states $S$ is small for $\mathrm{HI}$ and $\mathrm{HBr}$, but large and negative for $\mathrm{HCl}$ (i.e. there is a strong preference for $\mathrm{H}$-end excitation). For transitions to $\Omega^{\prime}=1.5$ for all collision partners $S$ is more positive for $e$ final $\Lambda$-doublet states than for $f$ final states. The larger steric asymmetry for $\mathrm{HI}$ in $e$ states compared to $\mathrm{HCl}$ is attributed to the larger polarizability and electric quadrupole of the HI molecule. The information gained from the integral steric asymmetry can also be rationalized in terms of loss mechanisms via reactive paths, involving $\mathrm{H}^{\prime}$ migration or an exchange reaction. ${ }^{99}$ Furthermore, the differences in the potentials for each HX may be probed using the integral steric asymmetry: for example, for $\mathrm{HCl}$ the global minimum of the PES is very deep, leading to a large anisotropy and a preference for the O-end to sample the well region, and to a negative value of $S$ (for $\left|\Omega^{\prime}=1.5, f\right\rangle$ ). ${ }^{99}$ The differences observed between $\mathrm{X}=\mathrm{I}$, and $\mathrm{Br}$ and $\mathrm{X}=\mathrm{Cl}$ do not currently have a simple explanation.

The effect of $\mathrm{OH}(\mathrm{X})$ orientation on reactive collisions of $\mathrm{OH}$ with $\mathrm{HBr}$ has recently been investigated by Tsai et al. ${ }^{102,103}$ Orientation of the $\mathrm{OH}$ was achieved using a hexapole electric field for state selection, combined with a static electric field for orientation as with previous experiments. The $\mathrm{Br}$ atom formed in the reaction was probed using VUV laser induced fluorescence detection. A strong preference for $\mathrm{O}$ end attack was observed, ${ }^{102}$ however $\mathrm{H}$ end attack also showed some reactivity. By comparison, the reactivity of side on attack was found to be much smaller. ${ }^{103}$ Two reaction pathways were suggested: collisions with the $\mathrm{O}$ end of the molecule result in direct $\mathrm{H}$ atom abstraction from the $\mathrm{HBr}$, whereas $\mathrm{H}$ end attack may involve $\mathrm{H}$-atom migration from the $\mathrm{H}-\mathrm{Br} .{ }^{103}$

Whilst most integral steric asymmetry measurements explore the collisions of open-shell molecules with closed-shell atoms or molecules, the number of collision partners has increased dramatically over the last decade. The inelastic collisions of oriented $\mathrm{OH}(v=0, \Omega=1.5, j=1.5, f)$ with $\mathrm{CO}$ and $\mathrm{N}_{2}$ has been used to probe systematic differences between the collision partners, particularly the symmetry of their interaction potentials, as well as the influence of complex formation on the collision process. ${ }^{75}$ The effect of molecular alignment of metastable $\mathrm{CO}\left(\mathrm{a}^{3} \Pi_{1}\right)$ (created using optical electronic excitation of $\operatorname{CO}\left(X^{1} \Sigma^{+}, v=0, J=0\right)$ using linearly polarized light) with NO has also been investigated. ${ }^{104}$ The dependence of the steric asymmetry on the repulsive part of the interaction potential has also been probed by comparing the collision of NO with $\mathrm{D}_{2}$ versus He. For spin-orbit conserving collisions, the two systems were found to have similar trends in the integral steric asymmetry with $\Delta j .{ }^{105}$ 


\subsection{Differential steric asymmetry}

Deeper insight into the dynamics of an inelastic or reactive collision can be obtained from measurements of the differential steric asymmetry, which quantifies the steric preference of the collision as a function of scattering angle of the products. For many years such measurements have been performed for reactive systems, providing valuable information on the underlying mechanism and dynamics of the reaction. The first such experiments were performed in the groups of Brooks and Bernstein in the 1970s. ${ }^{106,107}$ These early experiments have been reviewed extensively elsewhere, ${ }^{2,70,108}$ and so will not be discussed further here.

The first experiments to investigate the effect of alignment of a molecule before a reactive collision on the angular distribution of the products were performed by Loesch et al. ${ }^{109-111}$ on the $\mathrm{Li}+\mathrm{HF}(v=1)$ $\rightarrow \mathrm{LiF}+\mathrm{H}$ reaction, and later on the $\mathrm{Sr}+\mathrm{HF}(v=1) \rightarrow \mathrm{SrF}+\mathrm{H} \cdot{ }^{95,96}$ The aligned distribution of the $\mathrm{HF}$ bond axis was achieved using optical excitation with linearly polarized light to excite the $v^{\prime}=1 \leftarrow v=0$, $R(1), \Delta m=0$ transition of HF molecules in the $|v j m\rangle=|110\rangle$ state. A static electric field was employed to avoid depolarization of the aligned distribution via nuclear spin-rotation depolarization. The angular distribution of the products was measured using a rotatable surface ionization detector. The integral steric effect for collisions of aligned HF with Li was found to be relatively small, with a weak preference for the bond axis distribution to be aligned along the relative velocity. However, the DCSs and product velocity distributions for different alignments of the initial bond axis distribution, achieved by changing the direction of the light polarization with respect to the relative velocity, shown in Fig. 7, were found to be significantly different. ${ }^{112}$ It was seen that end-on collisions resulted in decreased forwards scattering and increased backwards scattering compared with side-on collisions, and an isotropic bond axis distribution. The propensity for end-on collisions to result in backwards scattered products was explained as follows. Although the distribution of the molecular bond axis is aligned, as opposed to oriented, only collisions with the F-end of the HF molecule result in reactions. After the collision of the Li with the F-end of the molecule, the $\mathrm{H}$ atom will be ejected along the HF axis into the forwards scattered direction, resulting in backwards scattered LiF. ${ }^{110}$ QCT-calculated product velocity distributions are also shown in Fig. 7. High recoil velocities are favoured when the bond axis distribution is aligned along the $x$ axis, whereas lower recoil velocities are favoured for collisions in which the alignment is along the relative velocity $(z$ direction). This effect shows that the distribution of final product states is sensitive to the initial alignment 
of the HF bond axis. ${ }^{112}$ Fig. 7 displays experimental laboratory frame angular distributions for the $\mathrm{Li}+\mathrm{HF}$ reaction compared with QCT calculations. ${ }^{112}$ The agreement between experimental and QCT calculations was found to be very good.

Further experiments by Loesch et al. used the brute force orientation method to achieve oriented distributions of the ICl diatomic molecule ${ }^{48}$ and various R-I hydrocarbon ${ }^{45,113,114}$ and aromatic molecules. ${ }^{49,50}$ The $\mathrm{K}+\mathrm{ICl}$ reaction was found to yield both the $\mathrm{KCl}$ and $\mathrm{KI}$ reaction products. Surprisingly, differential steric preference measurements indicated that the $\mathrm{KCl}$ product was preferentially formed from collisions of the $\mathrm{K}$ atom with the I-end of the $\mathrm{ICl}$ product, and similarly that $\mathrm{KI}$ was formed predominantly from collisions with the $\mathrm{Cl}$-end of the molecule. ${ }^{48}$ The reaction was found to proceed via a harpoon mechanism, resulting in forwards scattered products, and the counter-intuitive steric asymmetry results were interpreted in terms of a simple direct-interaction-with-product-repulsion (DIPR) model. ${ }^{48}$

Investigation of the $\mathrm{K}+\mathrm{I}-\mathrm{R}(\mathrm{R}=$ iodobenzene, 2-,3- and 4-iodotoluene) by Loesch et al. reaction found a strong differential steric preference. ${ }^{49,50}$ The differential steric effect for the bond axis parallel to the relative velocity indicated a preference for collisions at the R-end of the molecule for forwards scattered products and I-end attack for backwards scattered products. Overall, it was seen that I-end attack was most favourable for product formation, suggesting a K-I-R transition state. The perpendicular steric effect was investigated by directing the electric field perpendicular to the relative velocity, which provided information on whether products have a propensity to be scattered parallel the C-I bond (from C to I) or antiparallel after sideways attack. It was found that the perpendicular steric effect was positive over the entire angular range, indicating that the reaction products were predominantly scattered parallel to the C-I bond. These observations were rationalized using a cone of ejection model. At a critical distance, the electron from the $\mathrm{K}$ atom jumps to the $\mathrm{R}-\mathrm{I}$ molecule resulting in a repulsive anionic state. The anionic state rapidly dissociates and the $\mathrm{K}^{+}$and $\mathrm{I}^{-}$products rebound. However, unlike in the DIPR model, ${ }^{49,115}$ the departing ions interact with the $\mathrm{R}$ fragment and the products appear within a cone of ejection. ${ }^{50}$ Similar results were seen for the $\mathrm{K}+i-\mathrm{C}_{3} \mathrm{H}_{7}$ and $t-\mathrm{C}_{4} \mathrm{H}_{9}$ reactive systems. ${ }^{114}$

Optical alignment methods have also been used to investigate the differential steric effect in the $\mathrm{Cl}$ $+\mathrm{CHD}_{3}(v=1)$ and $\mathrm{CH}_{4}(v=1)$ systems. Initial experiments by Simpson et al. found a marked steric effect, ${ }^{23}$ indicating that collisions in which $\mathrm{Cl}$ atoms approach the $\mathrm{C}-\mathrm{H}$ stretch perpendicularly result in preferentially forwards scattered products. Investigations of the unpolarized $\mathrm{O}+\mathrm{CDH}_{3}$ and $\mathrm{F}+\mathrm{CHD}_{3}$ systems found evidence of significant reorientation effects of the molecule en route to the reaction bar- 
rier. ${ }^{26,116,117}$ The observation of a significant steric effect in the reaction of aligned $\mathrm{CDH}_{3}$ and $\mathrm{Cl}$ therefore suggests that reorientation effects are not significant in this system, otherwise prealignment of the reagents would have little effect on the outcome of the reaction due to the strong steering interactions. A detailed review comparing "active" steric control, in which the reagents are prealigned experimentally, and "passive" steric control, in which significant reorientation of the reagents results in steering of the reactant molecule in such reactions, is given in Ref. [26], and so will not be repeated here.

A series of recent experiments performed in the Liu group ${ }^{24-26,118}$ have probed the differential steric preference of the $\mathrm{CD}_{3} \mathrm{H}+\mathrm{Cl}$ reaction. In these experiments, an aligned distribution of $\mathrm{CD}_{3} \mathrm{H}$ was created using linearly polarized IR radiation tuned to the $R(0)$ transitions of the $\mathrm{CD}_{3} \mathrm{H}\left(v_{1}=1 \leftarrow 0\right)$. Hyperfine depolarization of the aligned distribution was measured and accounted for in the analysis of the experimental images. The $\mathrm{HCl}$ product was detected using resonantly enhanced multi-photon ionization (REMPI) combined with time-sliced velocity-map ion imaging. A large differential steric preference was seen for the $\mathrm{HCl}(v=0)$ product, ${ }^{24}$ as shown in Fig. 8. Approach of the $\mathrm{Cl}$ in a linear geometry (aligned with parallel polarization of the IR laser) results in an overall larger cross section and increased backwards scattering. On the other hand, side-on approach of the $\mathrm{Cl}$ (perpendicular IR polarization) results in sideways scattered products with a much smaller reaction probability. These observations are in agreement with a direct reaction mechanism with a collinear transition state. The collinear approach results in backwards scattering from small impact parameter, end-on collisions whereas for side-on approach larger impact parameter collisions lead to sideways scattered products. The steric effect for the $\mathrm{HCl} v=1$ product is not as easy to interpret. It was suggested that the $\mathrm{HCl} v=1$ products could be formed via a time delay mechanism in which there is substantial reorientation of the $\mathrm{C}-\mathrm{H}$ bond axis prior to, or during, the collision. ${ }^{25}$

In order to fully unravel the three dimensional effect of the bond axis orientation, Wang et al. performed the experiment in eight different geometries and were able to extract the $S_{q+}^{\{2\}}(\theta) j$-PDDCSs described in Section 2. ${ }^{25,118}$ As discussed in Section 2, these quantities by definition do not depend on how the bond axis distribution was prepared, and describe only the intrinsic steric requirement of the reaction. $S_{0}^{0}(\theta)$ represents the angular distribution of the products, the $S_{0}^{\{2\}}(\theta)$ and $S_{2+}^{\{2\}}(\theta)$ PDDCSs describe the propensity for the reaction to require alignment of the $\mathrm{C}-\mathrm{H}$ bond along the $z$ axis, and the $x$ axis with respect to $y$, respectively, and $S_{1+}^{\{2\}}(\theta)$ quantifies the tilt of the C-H bond with respect to the $x y$ and $x z$ planes. For the physical interpretation of the $j$-PDDCSs, the renormalized quantities $S_{q}^{\{k\}}(\theta) / S_{0}^{0}(\theta)$ are 
shown in Fig. 9. The measured renormalized $j$-PDDCSs for $v=0$ are in agreement with a line-of-centres collision model, ${ }^{1}$ indicated by the solid line in Fig. 9. At backwards scattering angles, $\theta=180^{\circ}$, the value of $S_{0}^{\{2\}}(\theta) / S_{0}^{0}(\theta)$ is near its lower limit, indicating a strong preference for $\boldsymbol{j}^{\prime}$ perpendicular to $\boldsymbol{k}$, and therefore $r$ to be parallel to $k$, as depicted in the first cartoon at the bottom of Fig. 9. At sideways scattering $\left(\theta=90^{\circ}\right)$, the $S_{0}^{\{2\}}(\theta) / S_{0}^{0}(\theta)$ goes to zero, but the $S_{1+}^{\{2\}}(\theta) / S_{0}^{0}(\theta)$ moment nears its lower limit, such that $\boldsymbol{r}$ is parallel to $(x+z)$, as depicted in the second cartoon. As the scattering becomes increasingly forwards, the $S_{1+}^{\{2\}}(\theta) / S_{0}^{0}(\theta)$ goes to zero, and the $S_{0}^{\{2\}}(\theta) / S_{0}^{0}(\theta)$ and $S_{2+}^{\{2\}}(\theta) / S_{0}^{0}(\theta)$ moments take their upper and lower limiting values respectively suggesting alignment of the $\mathrm{C}-\mathrm{H}$ bond along the $x$ axis. It should be noted that whilst these cartoons depict orientation of $\mathrm{CD}_{3} \mathrm{H}$, experimentally the molecule is aligned, and so strictly speaking it is not possible to distinguish between 'heads' and 'tails' collisions.

Measurements of the differential steric asymmetry discussed so far have been limited to reactive scattering systems, and the observed differential steric preferences have been well-explained using classical models. In contrast, recent experiments on the differential steric asymmetry in rotationally inelastic collisions of $\mathrm{NO}(\mathrm{X})+$ Ar have found that that the steric preference cannot be explained classically, and is due to quantum interference effects. ${ }^{73}$ As in the case of the integral steric asymmetry measurements, ${ }^{57,71,76,79}$ in these experiments the $\mathrm{NO}(\mathrm{X})$ was prepared in the $|\Omega=0.5, j=0.5, f\rangle$ state using an inhomogeneous hexapole electric field. The state selected $\mathrm{NO}(\mathrm{X})$ was then oriented in the interaction region using a static electric field generated using a four rod arrangement, as described in Section 3.3. As discussed, this method of orientation of the $\mathrm{NO}(\mathrm{X})$ results in a coherent superposition of the field free $f$ and $e \Lambda$-doublet levels, as described by Eq. (26).

Fig. 10 displays experimental images for collisions of oriented NO with Ar. ${ }^{73}$ Differences in the experimental images are clear for all final rotational states. The bond orientation is not seen to have a significant effect of the number or position of the peaks in the experimental image, but does have a significant effect on the relative intensity of the peaks. Previous work has shown that the peak structure seen in the field-free DCSs can be associated with quantum interferences between trajectories impacting on the sides and two ends of the NO molecule. ${ }^{80}$ Fits to the experimental images are compared to QM calculations in Fig. 10 and the agreement can be seen to be good.

The differential steric asymmetry for $\mathrm{NO}+$ Ar collisions is defined as ${ }^{73}$

$$
\mathrm{d} \sigma_{\mathrm{diff}}=\frac{\mathrm{d} \sigma_{\mathrm{N}}-\mathrm{d} \sigma_{\mathrm{O}}}{\mathrm{d} \sigma_{\mathrm{N}}+\mathrm{d} \sigma_{\mathrm{O}}}
$$


where $\mathrm{d} \sigma_{\mathrm{i}}$ is the DCSs for collision at the $i$-end of the molecule. It is important to note that integration of Eq. (29) for the differential steric asymmetry, d $\sigma_{\text {diff }}$, does not yield the integral steric asymmetry, $S$, since the latter is obtained from the (separately integrated) integral cross sections. Fig. 11 compares the differential steric asymmetry obtained from the experimental measurements with those calculated from QM and QCT scattering methods. The classical calculations fail to predict the oscillations in the differential steric asymmetry that are observed for low and middle $j^{\prime}$ in the experimental measurements and in the QM calculations, predicting very little steric preference other than in the very forward scattered direction for these states. At high rotational excitation $(\Delta j=15)$, the experimental measurements and QM calculations are in agreement with the classical predictions. As with the integral steric asymmetry, this can be attributed to the $\mathrm{CM}$ of NO being slightly displaced towards the O-end of the molecule, such that collisions with the $\mathrm{N}$-end of the molecule are able to exert more torque on the molecule, and therefore an $\mathrm{N}$-end steric preference is observed for high rotational excitation. The oscillations observed in the experimental and QM differential steric asymmetry can be rationalized using a semi-classical four path model, adapted from a similar model used to predict the position of parity dependent oscillations in $\mathrm{NO}(\mathrm{X})+$ Ar DCSs. ${ }^{73,80}$ Within this model, the steric asymmetry arises from a quantum interference between scattering from the two ends of the NO molecules. ${ }^{73}$

\section{Conclusions and future prospects}

This Perspective has focussed on recent studies of the effects of initial bond axis and angular momentum polarization on inelastic and reactive scattering. We have outlined the theoretical foundations for such studies, and described some of the strategies available for experimentally interrogating dynamical stereochemical effects. Some examples of recent work in this field have also been highlighted. There is considerable interest at the moment in the alignment and orientation of molecules using intense fields ${ }^{38,43,119}$ and with use of coherent laser excitation, ${ }^{30,31}$ which we have only touched on in this article. If such methods of reactant polarization can be combined with techniques for controlling and cooling the translational motion of molecules, ${ }^{84,120-122}$ and for conformer selection, ${ }^{123}$ then we anticipate that many detailed features of the stereodynamics of molecular collisions will be revealed. The research described in this Perspective on the scattering dynamics of NO with the rare gases has highlighted the effects of quantum interference. Such quantum effects are likely to be more readily observed as the collision energy is 
lowered, and the experimental conditions become more tightly defined.

We have said little about the polarization of the reaction products, yet this topic is intimately linked to the orientation and alignment of the reactants. ${ }^{124-126}$ In the case of inelastic scattering, it is common to invoke conservation of the projection of $\boldsymbol{j}$ along the kinematic apse, where the apse is defined ${ }^{127,128}$

$$
\hat{a}=\frac{p^{\prime}-p}{\left|p^{\prime}-p\right|}
$$

where $p$ and $p^{\prime}$ are the initial and final relative linear momenta. This projection is a rigorously conserved in the limit of classical encounters of hard-shell particles. ${ }^{127,128}$ Studies of the rotational tilt - the $\boldsymbol{j}-\boldsymbol{j}^{\prime}$ correlation - indicate that the projection of $j$ along the apse can show a propensity to be conserved, even in fairly attractive, non-hard-shell systems. ${ }^{129-131}$ Similar line-of-centres type propensities may also operate in reactive systems. ${ }^{26,125}$

However, in quantum mechanics, the product rotational angular momentum orientation and alignment can both differ from that predicted by the kinematic apse model, even in the case of hard-shell encounters. ${ }^{89,132-134}$ Furthermore, it has been shown that the orientation of the product rotational angular momentum in inelastic scattering can be strongly influenced by QM interference effects. ${ }^{132,133}$

Fig. 12 shows experimentally determined product rotational orientation for collisions of $\mathrm{NO}(\mathrm{X})$ with $\mathrm{Kr}$, obtained with full initial and final $\Lambda$-doublet state resolution. Experimental DCS images were recorded using right $(\mathrm{R})$ and left $(\mathrm{L})$ handed circularly polarized light. The experimental DCS image obtained from summing these images is shown in panel a) of Fig. 12. Information on the rotational orientation of the NO after the collision can be obtained from the normalized difference of the R and $\mathrm{L}$ images, shown for the experimental data in the second column of panel a) of Fig. 12. The oscillations in intensity (color) in the difference images reflect the fact that for some scattering angles the NO product rotates clockwise in the plane of the image, whilst at other angles the scattered molecule rotates counterclockwise. ${ }^{132}$ The experimental normalized difference image is compared with simulations performed using the results from both QM and QCT calculations. It is clear that the QCT simulation fails to reproduced the oscillations between positive and negative intensity that can be observed in the experimental and QM simulations.

Panel b) of Fig. 12 compares the experimental, QM, and QCT angular distributions, normalized $n_{1-}^{\{1\}}(\theta) j^{\prime}$-PDDCS, and renormalized $\rho_{1-}^{\{1\}}(\theta) j^{\prime}$-PDDCS, analogous to the reactant $j$-PDDCS of Eq. (8). 
Unlike the $S_{q}^{\{k\}}(\theta) j$-PDDCSs introduced in Section 2.2, which describe the orientation or alignment of the reactant rotational angular momentum preferred for reaction, the $j^{\prime}$-PDDCSs displayed in Fig. 12 quantify the orientation of the rotational angular momentum of the scattered NO molecule after the collision. A positive value of these $j^{\prime}$-PDDCSs indicates a preference for $j^{\prime}$ to lie parallel to the scattering frame $y$-axis, whilst a negative value indicates that $j^{\prime}$ lies antiparallel to the $y$-axis. The renormalized $j^{\prime}$-PDDCS is given by dividing the normalized PDDCS by the angular distribution according to

$$
\rho_{1-}^{\{1\}}(\theta)=\frac{n_{1-}^{\{1\}}(\theta)}{n_{0}^{0}(\theta)}
$$

The agreement between the experimental, QM, and QCT angular distributions is generally good, with all three sets of data showing predominantly forwards scattered products as would be expected from glancing blow collisions that result in low rotational excitation. However, comparing the normalized $j^{\prime}$-PDDCSs, although it can be seen that the agreement between the experimental and QM data is good, large discrepancies between these two sets of data and the QCT calculations can be seen. In the QCT normalized $j^{\prime}$-PDDCS, the dominant feature is a positive peak in the forwards scattered direction, however this is not observed in the experimental or QM data, which oscillate around zero throughout the entire angular range. It is perhaps more instructive to consider the renormalized $j^{\prime}$-PDDCSs, shown in the far right of panel b) of Fig. 12. The QCT $j^{\prime}$-PDDCS is positive at small scattering angles and decreases relatively smoothly to become negative at scattering angles above $60^{\circ}$. In contrast, the experimental and QM data show near zero orientation at small scattering angles, and then oscillate between positive and negative orientation with scattering angle. This indicates that the experimental product rotational orientation is dominated by QM interference effects. ${ }^{132,133}$

The product rotational angular momentum orientation is likely to be highly sensitive to the initial bond axis orientation of the reactants, i.e. strong $\boldsymbol{r}-\boldsymbol{k}-\boldsymbol{j}^{\prime}-\boldsymbol{k}^{\prime}$ four-vector correlations are likely to be present in this system, and are likely to be highly revealing about the scattering dynamics in the $\mathrm{NO}+\mathrm{Kr}$ collision system. Similar orientation measurements in more complex inelastic and reactive scattering systems are also likely to be revealing, since the product rotational orientation should be very sensitive to the balance between impulsive, attractive, and complex-forming scattering dynamics.

Measurements of three and four vector correlations lift the constraint of azimuthal symmetry about the relative velocity vector inherent to measurements of the DCS, and open the way to investigate in 
detail the intimate mechanism of molecular collisions. Additionally, they provide the experimenter with a means to control the outcome of chemical reactions. Determination and interpretation of such effects would represent a new beginning in molecular reaction dynamics, and a realization of Herschbach and coworkers original dream of measuring four-vector correlations in molecular collisions and unveil how chemical reactions and inelastic collisions take place. $^{2-4}$

\section{Acknowledgements}

The support of the UK EPSRC (to M.B. via Programme Grants EP/G00224X/1 and EP/L005913/1), the EU (to M.B. via FP7 EU People ITN project 238671), and the Spanish Ministry of Science and Innovation (grants CTQ2012-37404 and CSD2009-00038, to FJA) are gratefully acknowledged. S.S. acknowledges support from the National Basic Research Program of China (973 program) under grant No. 2013CB922200, and from the National Science Foundation of China under grant Nos. 11034003 and 91221301. SDSG and MB also thank Cambio Ltd. and Dr Peter Dean for generous support. The authors would like to thank Marcelo de Miranda, Chris Eyles, Helen Chadwick, and Balazs Hornung for their important contributions to the work described in this Perspective.

\section{References}

[1] R. D. Levine and R. B. Bernstein, Molecular Reaction Dynamics and Chemical Reactivity, (Oxford University Press, New York, USA), 1987.

[2] R. B. Bernstein, D. R. Herschbach and R. D. Levine, J. Phys. Chem., 1987, 91, 5365-5377.

[3] D. A. Case and D. R. Herschbach, Mol. Phys., 1975, 30, 1537-1564.

[4] J. D. Barnwell, J. G. Loeser and D. R. Herschbach, J. Phys. Chem., 1983, 87, 2781-2786.

[5] K. Blum, Density matrix theory and applications, Springer Science \& Business Media, Heidelberg, Dordrecht, London, New York, 3rd edn., 2012, vol. 64.

[6] R. N. Zare, Angular momentum: understanding spatial aspects in chemistry and physics, WileyInterscience, 2013. 
[7] J. Mikosch, S. Trippel, C. Eichhorn, R. Otto, U. Lourderaj, J. X. Zhang, W. L. Hase, M. Weidemueller and R. Wester, Science, 2008, 319, 183-186.

[8] M. P. de Miranda, F. J. Aoiz, L. Banares and V. S. Rábanos, J. Chem. Phys., 1999, 111, 5368-5383.

[9] J. Aldegunde, M. P. de Miranda, J. M. Haigh, B. K. Kendrick, V. Sáez-Rábanos and F. J. Aoiz, J. Phys. Chem. A, 2005, 109, 6200-6217.

[10] M. Brouard and C. Vallance, Tutorials in molecular reaction dynamics, Royal Society of Chemistry, Cambridge, U.K., 2010.

[11] R. G. Newton, Texts and Monographs in Physics: Scattering theory of waves and particles, Springer, Berlin, Heidelberg, 1982.

[12] M. P. de Miranda and F. J. Aoiz, Phys. Rev. Lett., 2004, 93, 083201.

[13] M. P. de Miranda, F. J. Aoiz, V. Sáez-Rábanos and M. Brouard, J. Chem. Phys., 2004, 121, 98309843.

[14] I. V. Hertel and W. Stoll, Adv. At. and Mol. Phys., 1978, 13, 113-228.

[15] F. J. Aoiz, M. Brouard and P. A. Enriquez, J. Chem. Phys., 1996, 105, 4964-4982.

[16] J. Aldegunde, J. M. Alvariño, B. K. Kendrick, V. Sáez-Rábanos, M. P. de Miranda and F. J. Aoiz, Phys. Chem. Chem. Phys., 2006, 8, 4881-4896.

[17] A. J. Alexander, J. Chem. Phys., 2005, 123, 194312.

[18] M. P. de Miranda, S. D. S. Gordon and J. Aldegunde, Mol. Phys., 2012, 110, 1883-1892.

[19] R. E. Drullinger and R. N. Zare, J. Chem. Phys., 1969, 51, 5532-5542.

[20] R. Bersohn and S. H. Lin, Adv. Chem. Phys., 1969, 16, 67-100.

[21] R. N. Zare, Mol. Photochem., 1972, 4, 1-37.

[22] R. N. Zare, Berichte der Bunsengesellschaft für physikalische Chemie, 1982, 86, 422-425.

[23] W. R. Simpson, T. P. Rakitzis, S. A. Kandel, A. J. Orr-Ewing and R. N. Zare, J. Chem. Phys., 1995, 103, 7313-7335. 
[24] F. Wang, J. S. Lin and K. Liu, Science, 2011, 331, 900-903.

[25] F. Wang, K. Liu and T. P. Rakitzis, Nature Chemistry, 2012, 4, 636-641.

[26] K. Liu, J. Chem. Phys., 2015, 142, 080901.

[27] Z. Karny, R. C. Estler and R. N. Zare, J. Chem. Phys., 1978, 69, 5199-5201.

[28] M. J. Weida and C. S. Parmenter, J. Chem. Phys., 1997, 107, 7138-7147.

[29] S. A. Kandel, A. J. Alexander, Z. H. Kim, R. N. Zare, F. J. Aoiz, L. Bañares, J. F. Castillo and V. S. Rábanos, J. Chem. Phys., 2000, 112, 670-685.

[30] N. Mukherjee and R. N. Zare, J. Chem. Phys., 2010, 132, 154302.

[31] N. Mukherjee and R. N. Zare, J. Chem. Phys., 2011, 135, 024201.

[32] N. Mukherjee, W. Dong, J. A. Harrison and R. N. Zare, J. Chem. Phys., 2013, 138, 051101.

[33] W. Dong, N. Mukherjee and R. N. Zare, J. Chem. Phys., 2013, 139, 074204.

[34] K. Bergmann, H. Theuer and B. W. Shore, Rev. Mod. Phys., 1998, 70, 1003-1025.

[35] W. Kim and P. M. Felker, J. Chem. Phys., 1996, 104, 1147-1150.

[36] H. Sakai, C. P. Safvan, J. J. Larsen, K. M. Hilligso, K. Hald, H. Stapelfeldt et al., J. Chem. Phys., 1999, 110, 10235-10238.

[37] B. Friedrich and D. R. Herschbach, J. Phys. Chem. A, 1999, 103, 10280-10288.

[38] T. Seideman, J. Chem. Phys., 1995, 103, 7887-7896.

[39] H. Sakai, S. Minemoto, H. Nanjo, H. Tanji and T. Suzuki, Phys. Rev. Lett., 2003, 90, 083001.

[40] L. Holmegaard, J. H. Nielsen, I. Nevo, H. Stapelfeldt, F. Filsinger, J. Küpper and G. Meijer, Phys. Rev. Lett., 2009, 102, 023001.

[41] O. Ghafur, A. Rouzée, A. Gijsbertsen, W. K. Siu, S. Stolte and M. J. Vrakking, Nature Physics, 2009, 5, 289-293. 
[42] E. Hamilton, T. Seideman, T. Ejdrup, M. D. Poulsen, C. Z. Bisgaard, S. S. Viftrup and H. Stapelfeldt, Phys. Rev. A, 2005, 72, 043402.

[43] H. Stapelfeldt and T. Seideman, Rev. Mod. Phys, 2003, 75, 543-557.

[44] H. J. Loesch and A. Remscheid, J. Chem. Phys., 1990, 93, 4779-4790.

[45] H. J. Loesch and A. Remscheid, J. Phys. Chem., 1991, 95, 8194-8200.

[46] B. Friedrich and D. R. Herschbach, Nature, 1991, 353, 412—414.

[47] B. Friedrich, D. P. Pullman and D. R. Herschbach, J. Phys. Chem., 1991, 95, 8118-8129.

[48] H. J. Loesch and J. Möller, J. Chem. Phys., 1992, 97, 9016-9030.

[49] H. J. Loesch and J. Möller, J. Phys. Chem. A, 1997, 101, 7534-7543.

[50] H. Loesch and J. Möller, Faraday Disc., 1999, 113, 241-260.

[51] H. K. Hughes, Phys. Rev., 1949, 76, 1675.

[52] H. G. Bennewitz, W. Paul and C. Schlier, Zeitschrift für Physik A, 1955, 141, 6-15.

[53] K. H. Kramer and R. B. Bernstein, J. Chem. Phys., 1965, 42, 767-770.

[54] P. R. Brooks, Science, 1976, 193, 11-16.

[55] J. Bulthuis, J. Van Leuken, F. Van Amerom and S. Stolte, Chem. Phys. Lett., 1994, 222, 378-386.

[56] P. R. Brooks and E. M. Jones, J. Chem. Phys., 1966, 45, 3449-3450.

[57] J. van Leuken, J. Bulthuis, S. Stolte and J. Snijders, Chem. Phys. Lett., 1996, 260, 595-603.

[58] M. J. L. de Lange, S. Stolte, C. A. Taatjes, J. Kłos, G. C. Groenenboom and A. van der Avoird, J. Chem. Phys., 2004, 121, 11691-11701.

[59] A. Rouzée, A. Gijsbertsen and M. J. J. Vrakking, in Progress in Ultrafast Intense Laser Science VI, Springer, 2010, pp. 45-73.

[60] R. J. Beuhler Jr, R. B. Bernstein and K. H. Kramer, J. Am. Chem. Soc., 1966, 88, 5331-5332. 
[61] J. J. Everdij, A. Huijser and N. F. Verster, Rev. Sci. Instrum., 1973, 44, 721-725.

[62] E. M. Jones and P. R. Brooks, J. Chem. Phys., 1970, 53, 55-58.

[63] S. Stolte, J. Reuss and H. L. Schwartz, Physica, 1972, 57, 254-266.

[64] A. Rouzée, A. Gijsbertsen, O. Ghafur, O. M. Shir, T. Bäck, S. Stolte and M. J. Vrakking, New J. Phys., 2009, 11, 105040.

[65] J. A. Kerr, K P Huber and G. Herzberg, Molecular spectra and molecular structure: IV constants of diatomic molecules: Von NostrandReinhold, New York, 1979, pp. 716, 1982.

[66] M. H. Alexander, J. Chem. Phys., 1982, 76, 5974-5988.

[67] M. H. Alexander, Chem. Phys., 1985, 92, 337-344.

[68] P. J. Dagdigian, M. H. Alexander and K. Liu, J. Chem. Phys., 1989, 91, 839-848.

[69] M. H. Alexander, J. Chem. Phys., 1993, 99, 7725-7738.

[70] D. H. Parker and R. B. Bernstein, Ann. Rev. Phys. Chem., 1989, 40, 561-595.

[71] M. H. Alexander and S. Stolte, J. Chem. Phys., 2000, 112, 8017-8026.

[72] M. C. van Beek, J. J. ter Meulen and M. H. Alexander, J. Chem. Phys., 2000, 113, 637-646.

[73] B. Nichols, H. Chadwick, S. D. S. Gordon, C. J. Eyles, B. Hornung, M. Brouard, M. H. Alexander, F. J. Aoiz, A. Gijsbertsen and S. Stolte, Chem. Sci., 2015, 6, 2202-2210.

[74] M. C. van Beek, G. Berden, H. L. Bethlem and J. J. ter Meulen, Phys. Rev. Lett., 2001, 86, 40014004.

[75] M. C. van Beek and J. J. ter Meulen, J. Chem. Phys., 2001, 115, 1843-1852.

[76] M. J. L. de Lange, M. Drabbels, P. T. Griffiths, J. Bulthuis, S. Stolte and J. G. Snijders, Chem. Phys. Lett., 1999, 313, 491-498.

[77] A. T. J. B. Eppink and D. H. Parker, Rev. Sci. Instrum., 1997, 68, 3477-3484.

[78] D. W. Chandler and P. L. Houston, J. Chem. Phys., 1987, 87, 1445-1447. 
[79] A. Gijsbertsen, H. Linnartz, C. A. Taatjes and S. Stolte, J. Am. Chem. Soc., 2006, 128, 8777-8789.

[80] C. J. Eyles, M. Brouard, C.-H. Yang, J. Kłos, F. J. Aoiz, A. Gijsbertsen, A. E. Wiskerke and S. Stolte, Nature Chemistry, 2011, 3, 597-602.

[81] C. J. Eyles, M. Brouard, H. Chadwick, B. Hornung, B. Nichols, C.-H. Yang, J. Kłos, F. J. Aoiz, A. Gijsbertsen, A. E. Wiskerke and S. Stolte, Physical Chemistry Chemical Physics, 2012, 14, 5403-5419.

[82] G. Sarma, S. Marinakis, J. J. ter Meulen, D. H. Parker and K. G. McKendrick, Nature Chemistry, 2012, 4, 985-989.

[83] O. Tkáč, A. K. Saha, J. Onvlee, C.-H. Yang, G. Sarma, C. K. Bishwakarma, S. Y. T. van de Meerakker, A. van der Avoird, D. H. Parker and A. J. Orr-Ewing, Physical Chemistry Chemical Physics, 2014, 16, 477-488.

[84] M. Brouard, D. H. Parker and S. Y. T. van de Meerakker, Chem. Soc. Rev., 2014, 43, 7279-7294.

[85] M. Brouard, H. Chadwick, S. D. S. Gordon, B. Hornung, B. Nichols, J. Kłos, F. J. Aoiz and S. Stolte, J. Chem. Phys., 2014, 141, 164306.

[86] S. N. Vogels, J. Onvlee, A. von Zastrow, G. C. Groenenboom, A. van der Avoird and S. Y. T. van de Meerakker, Phys. Rev. Lett., 2014, 113, year.

[87] A. von Zastrow, J. Onvlee, S. N. Vogels, G. C. Groenenboom, A. van der Avoird and S. Y. van de Meerakker, Nature Chemistry, 2014, 6, 216-221.

[88] J. Onvlee, S. N. Vogels, A. von Zastrow, D. H. Parker and S. Y. van de Meerakker, Phys. Chem. Chem. Phys., 2014, 16, 15768-15779.

[89] H. Chadwick, B. Nichols, S. D. S. Gordon, B. Hornung, E. Squires, M. Brouard, J. Klos, M. H. Alexander, F. Javier Aoiz and S. Stolte, J. Phys. Chem. Lett., 2014, 5, 3296-3301.

[90] G. Sarma, A. K. Saha, J. J. ter Meulen, D. H. Parker and S. Marinakis, J. Chem. Phys., 2015, 142, 034309.

[91] H. Ito, M. Okada, D. Yamazaki and T. Kasai, J. Phys. Chem. A, 2010, 114, 3080-3086. 
[92] M. Hashinokuchi, M. Okada, H. Ito, T. Kasai, K. Moritani and Y. Teraoka, Phys. Rev. Lett., 2008, 100, 256104.

[93] D. H. Parker, K. K. Chakravorty and R. B. Bernstein, Chem. Phys. Lett., 1982, 86, 113-117.

[94] S. E. Choi and R. B. Bernstein, J. Chem. Phys., 1985, 83, 4463-4469.

[95] H. J. Loesch and F. Stienkemeier, J. Chem. Phys., 1994, 100, 740-743.

[96] H. J. Loesch and F. Stienkemeier, J. Chem. Phys., 1994, 100, 4308-4315.

[97] M. H. Alexander, J. Chem. Phys., 1999, 111, 7426-7434.

[98] A. Gijsbertsen, H. Linnartz, J. Kłos and S. Stolte, Phys. Scr., 2005, 72, C1-C5.

[99] A. Moise, D. H. Parker and J. J. ter Meulen, J. Chem. Phys., 2007, 126, 124302.

[100] R. Cireasa, A. Moise and J. J. ter Meulen, J. Chem. Phys., 2005, 123, 64310.

[101] A. Moise, R. S. Cireasa, D. H. Parker and J. J. ter Meulen, J. Chem. Phys., 2006, 125, 204315.

[102] P.-Y. Tsai, D.-C. Che, M. Nakamura, K.-C. Lin and T. Kasai, Phys. Chem. Chem. Phys., 2010, 12, $2532-2534$.

[103] P.-Y. Tsai, D.-C. Che, M. Nakamura, K.-C. Lin and T. Kasai, Phys. Chem. Chem. Phys., 2011, 13, $1419-1423$.

[104] M. Nakamura, D. C. Che, P. Y. Tsai, K. C. Lin and T. Kasai, J. Phys. Chem. A, 2013, 117, 81578162.

[105] C. A. Taatjes, A. Gijsbertsen, M. J. L. de Lange and S. Stolte, J. Phys. Chem. A, 2007, 111, 76317639.

[106] G. Marcelin and P. R. Brooks, J. Am. Chem. Soc., 1973, 95, 7885-7886.

[107] R. J. Beuhler Jr and R. B. Bernstein, J. Chem. Phys., 1969, 51, 5305-5315.

[108] H. J. Loesch, Ann. Rev. Phys. Chem., 1995, 46, 555-594.

[109] H. J. Loesch, E. Stenzel and B. Wüstenbecker, J. Chem. Phys., 1991, 95, 3841-3842. 
[110] H. J. Loesch and F. Stienkemeier, J. Chem. Phys., 1993, 98, 9570-9584.

[111] O. Höbel and H. J. Loesch, Faraday Discuss. Chem. Soc., 1999, 113, 337-338.

[112] F. J. Aoiz, M. T. Martınez and V. S. Rábanos, J. Chem. Phys., 2001, 114, 8880-8896.

[113] J. J. van Leuken, J. Bulthuis, S. Stolte and H. J. Loesch, J. Phys. Chem., 1995, 99, 13582-13590.

[114] H. J. Loesch and J. Möller, J. Phys. Chem. A, 1998, 102, 9410-9419.

[115] P. J. Kuntz, Trans. Farad. Soc., 1970, 66, 2980-2996.

[116] F. Wang and K. Liu, Chemical Science, 2010, 1, 126-133.

[117] W. Zhang, H. Kawamata and K. Liu, Science, 2009, 325, 303-306.

[118] F. Wang, J. S. Lin and K. Liu, J. Chem. Phys., 2014, 140, 084202.

[119] B. Friedrich and D. R. Herschbach, Phys. Rev. Lett., 1995, 74, 4623-4626.

[120] M. T. Bell and T. P. Softley, Mol. Phys., 2009, 107, 99-132.

[121] A. B. Henson, S. Gersten, Y. Shagam, J. Narevicius and E. Narevicius, Science, 2012, 338, $234-$ 238

[122] B. Bertsche, J. Jankunas and A. Osterwalder, Chimia, 2014, 68, 256-259.

[123] Y.-P. Chang, K. Dlugolecki, J. Kuepper, D. Roesch, D. Wild and S. Willitsch, Science, 2013, 342, $98-101$.

[124] S. K. Kim and D. R. Herschbach, Faraday Discuss., 1987, 84, 159-169.

[125] A. J. Orr-Ewing and R. N. Zare, Ann. Rev. Phys. Chem., 1994, 45, 315-366.

[126] M. Brouard, P. O’Keeffe and C. Vallance, J. Phys. Chem. A, 2002, 106, 3629-3641.

[127] V. Khare, D. J. Kouri and D. K. Hoffman, J. Chem. Phys., 1981, 74, 2275.

[128] D. K. Hoffman, J. W. Evans and D. J. Kouri, J. Chem. Phys., 1984, 80, 144.

[129] M. L. Costen, S. Marinakis and K. G. McKendrick, Chem. Soc. Rev., 2008, 37, 732. 
[130] G. Paterson, M. L. Costen and K. G. McKendrick, Int. Rev. Phys. Chem., 2012, 31, 69-109.

[131] H. Chadwick, M. Brouard, T. Perkins and F. J. Aoiz, Int. Rev. Phys. Chem., 2014, 33, 79-123.

[132] K. T. Lorenz, D. W. Chandler, J. W. Barr, W. Chen, G. L. Barnes and J. I. Cline, Science, 2001, 293, 2063-2066.

[133] M. Brouard, B. Hornung and F. J. Aoiz, Phys. Rev. Lett., 2013, 111, 183202.

[134] M. Brouard, H. Chadwick, C. J. Eyles, B. Hornung, B. Nichols, F. J. Aoiz, P. G. Jambrina, S. Stolte and M. P. de Miranda, J. Chem. Phys., 2013, 138, 104309. 


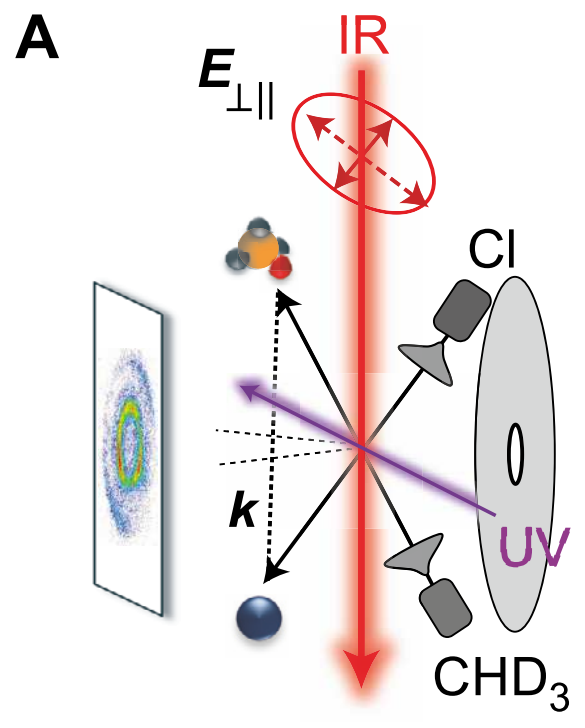

B

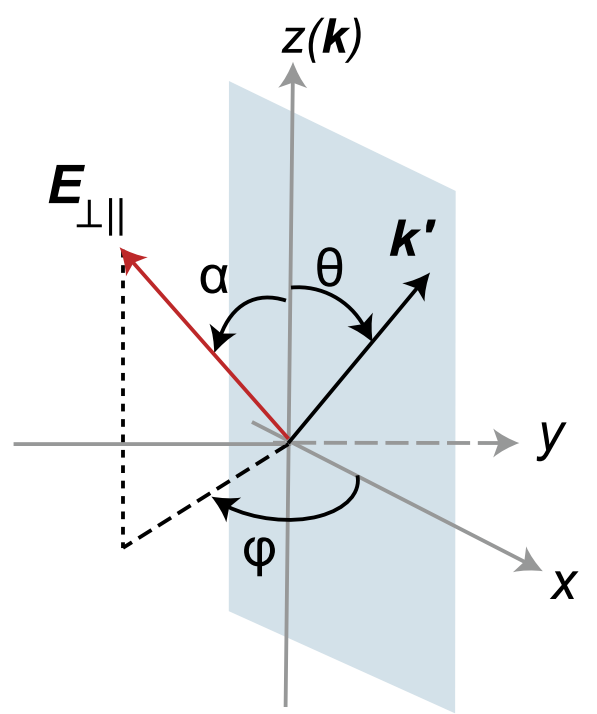

Figure 1: (A) The experimental setup used for the measurement of polarisation dependent differential cross sections. An infrared laser excites the $\mathrm{CHD}_{3}$. The polarisation of the infrared laser can be modified which causes the excited $\mathrm{CHD}_{3}$ to become polarised. A UV probe then ionises the $\mathrm{CHD}_{3}$ after reaction where it is mapped onto the detector. (B) The coordinate system used to describe the experiment. $E_{\perp \|}$ is the infrared laser polarisation at an angle $\alpha$ to the relative velocity. $\theta$ is the scattering angle and $\phi$ is the azimuthal component. Figure adapted from Ref. [25]. 


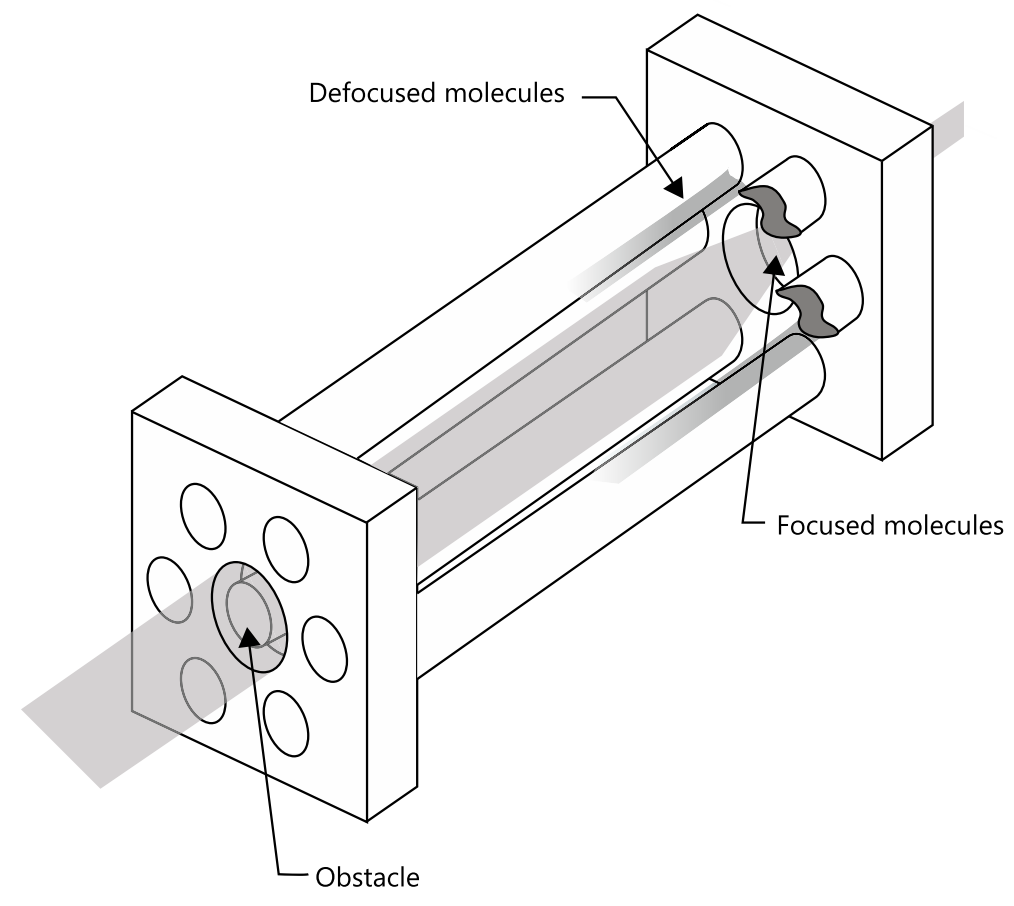

Figure 2: The hexapole state-selection apparatus consists of six alternately charged rods. The molecules enter the hexapole electric field and undergo a half wave of harmonic motion, focussing them into an interaction region. High field seeking states, those with which the energy is lowered by the Stark field are ejected from the hexapole. Low field seeking states whose energies are increased by being in the field are focussed into the centre by the Stark field. 


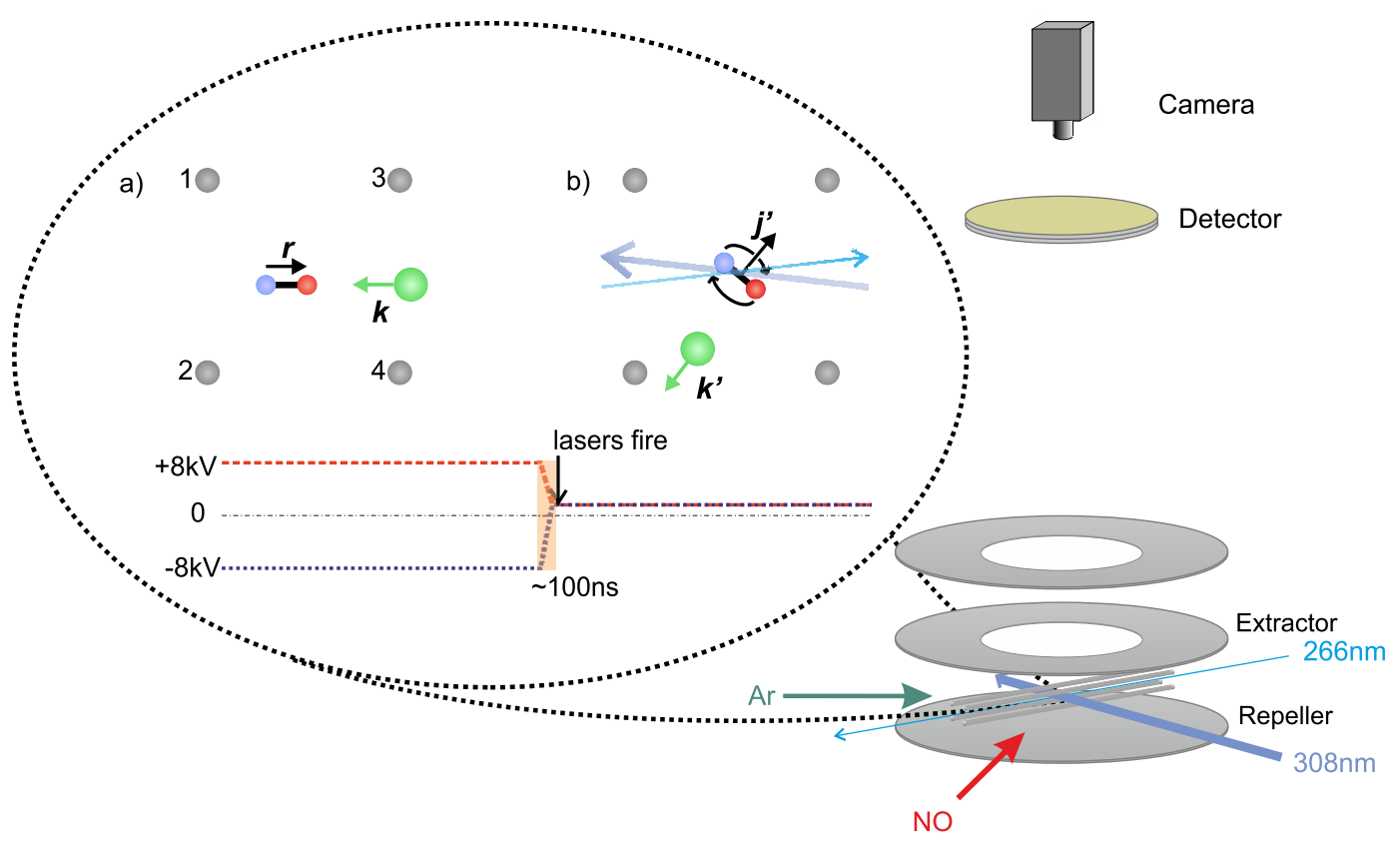

Figure 3: Schematic of the setup which allows imaging of steric effects, in this case for the scattering of $\mathrm{NO}+$ Ar. Panel (a) shows an expanded detail of the four-rod assembly, with the collision system just before collision. Panel (b) shows the system just after collision when the rods have been switched from orientation to velocity mapping conditions, as illustrated by the timing profile below. Also shown are the four vectors necessary to fully describe the collision process. Adapted from Ref. [73]. 


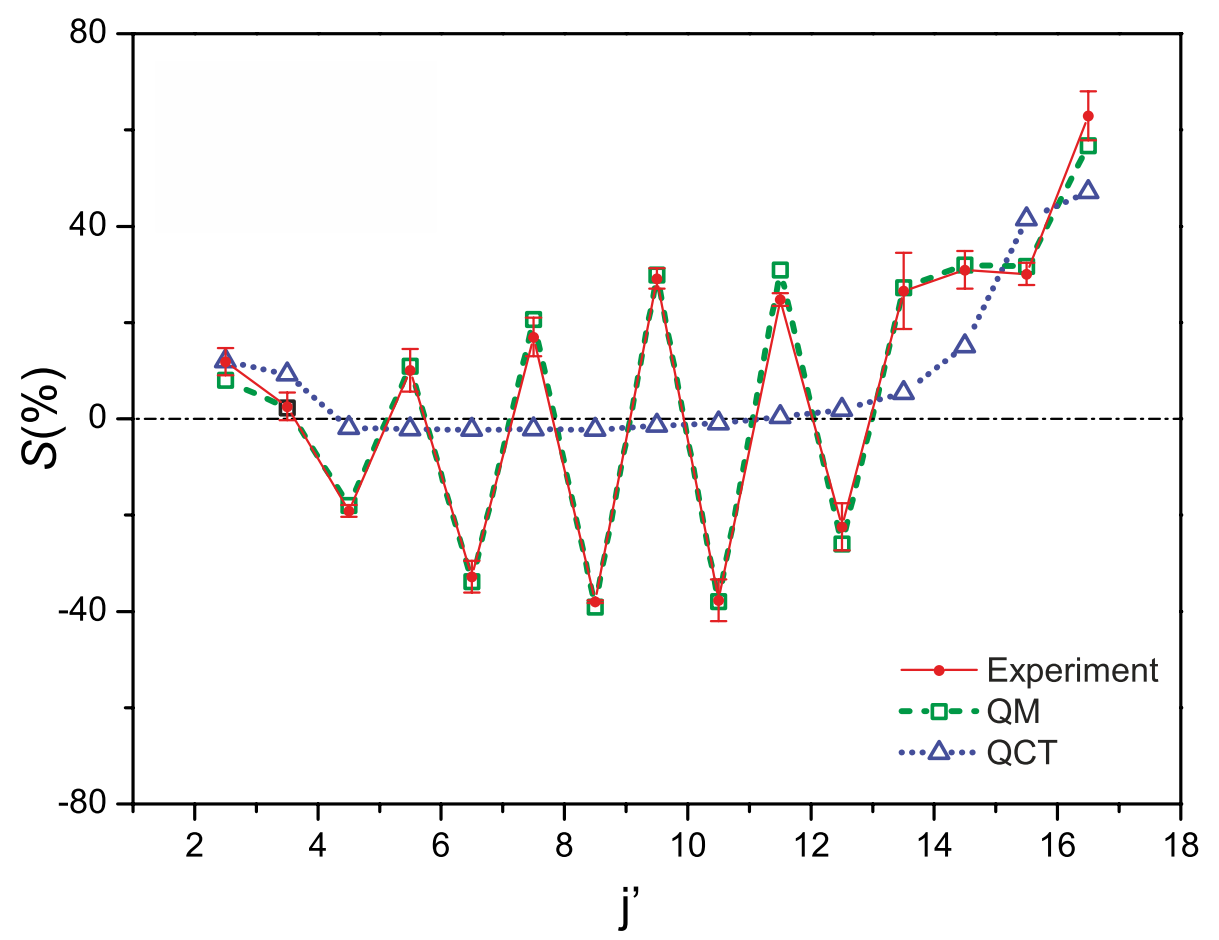

Figure 4: Comparison of experimental (red line with circles), QM (black dashed line with open squares), and QCT (blue dotted line with triangles) integral steric asymmetry for the inelastic scattering of $\operatorname{NO}\left(X^{2} \Pi, v=0, j=1 / 2, \Omega=1 / 2\right)$ by Ar. The integral steric asymmetry is defined in Eq. (28) of the main text, whereby a positive value of $S$ shows a preference for an $\mathrm{N}$-end collision. Figure adapted from Ref. [73]. 

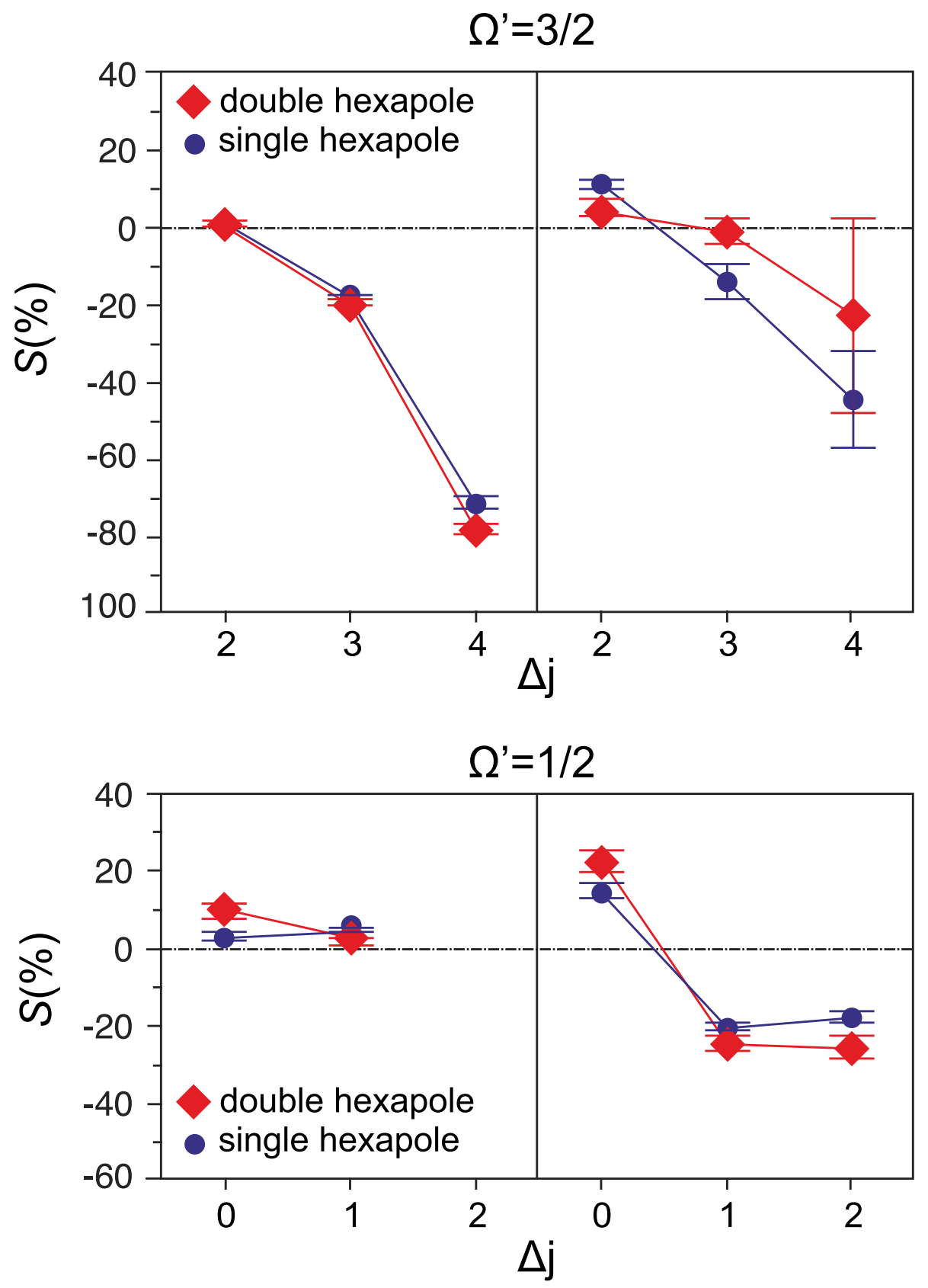

Figure 5: Comparison of the integral steric asymmetry for scattering of $\mathrm{OH}\left(X^{2} \Pi, v=0, \Omega=1.5, j=1.5\right)$ with Ar measured with single (red circles) and double (diamond) hexapole state selection. The left and right panels show scattering into $e$ and $f$ final $\Lambda$-doublet levels, respectively. Figure adapted from Ref. [ 72]. 

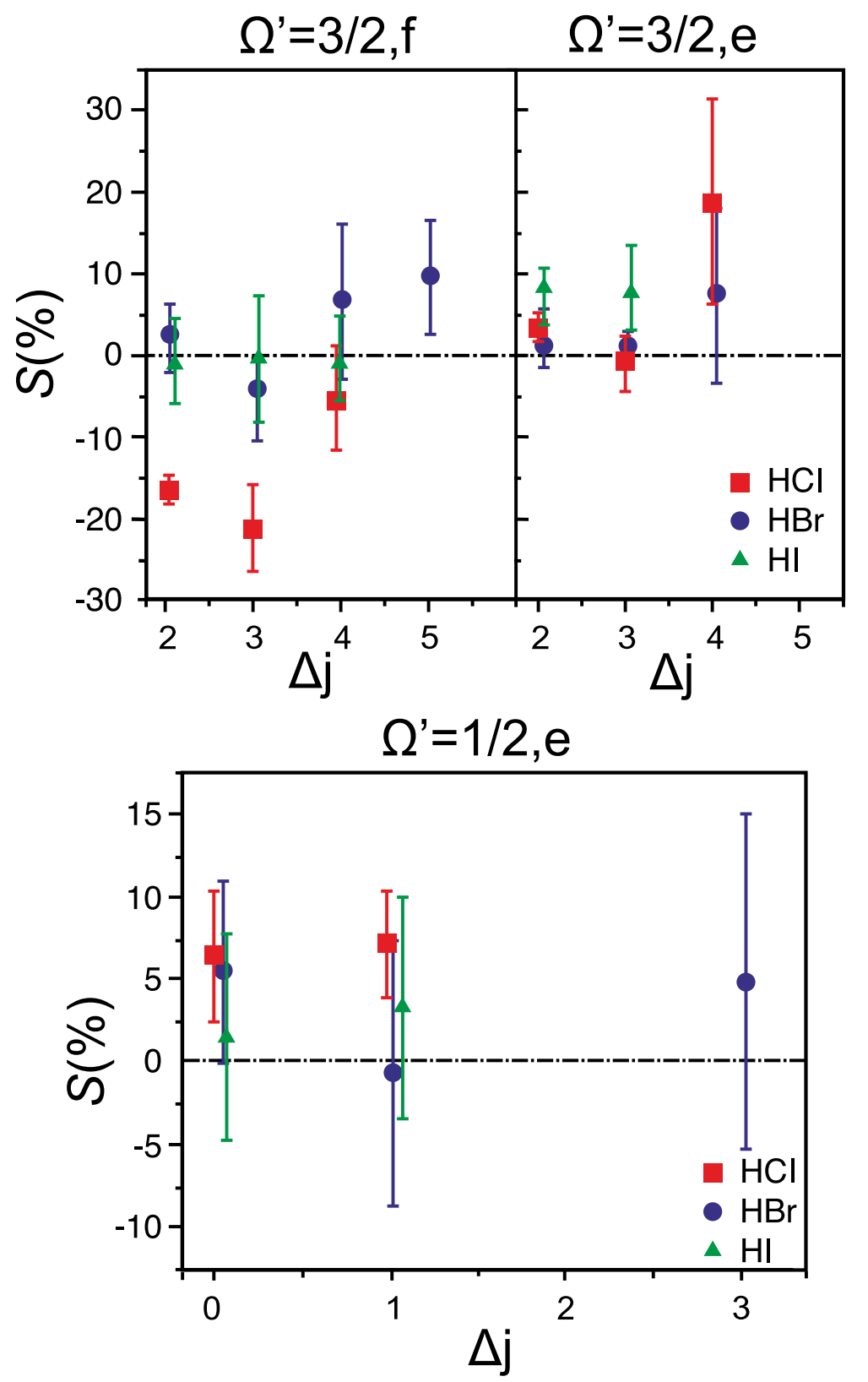

Figure 6: Integral steric asymmetry, $S$ for scattering of $\mathrm{OH}\left(X^{2} \Pi, v=0, \Omega=1.5, j=1.5\right)$ by $\mathrm{HI}$ at a collision energy of $690 \mathrm{~cm}^{-1}$ (green triangles), $\mathrm{HBr}$ at a collision energy of $750 \mathrm{~cm}-1$ (blue circles), and $\mathrm{HCl}$ at a collision energy of $920 \mathrm{~cm}^{-1}$ (red squares) for (top) spin-orbit conserving $\Omega^{\prime}=1.5$ and (bottom) spin-orbit changing $\Omega^{\prime}=0.5$ transitions. Figure adapted from Ref. [99]. 

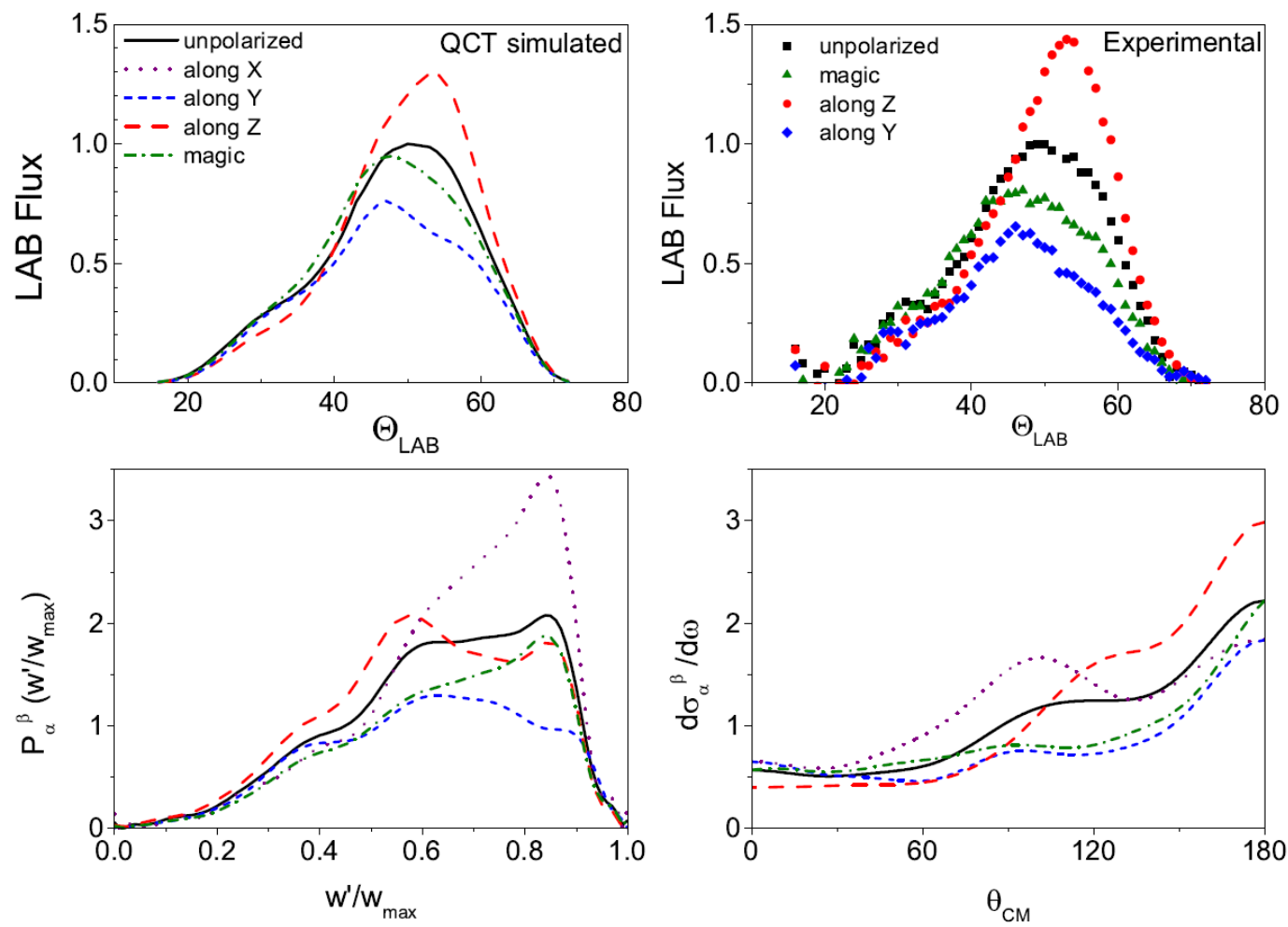

Figure 7: Experimental (right) and simulated (using the results of QCT calculations) laboratory frame angular distributions of scattered $\mathrm{LiF}$ after reactive collisions of $\mathrm{Li}+\mathrm{HF}(v=1)$. The data are shown for alignment of the bond axis distribution parallel to the $z$ axis, or relative velocity (red dashed line), $y$ axis (blue dotted line), $x$ axis (purple dotted) line, magic angle (green dash dot line) and an isotropic bond axis distribution (solid black line). In the bottom left panel, the QCT calculated product recoil distribution for different bond axis alignments is displayed. Corresponding QCT calculated differential cross sections for the collision are displayed in the bottom right panel. Adapted from Ref. [112]. 

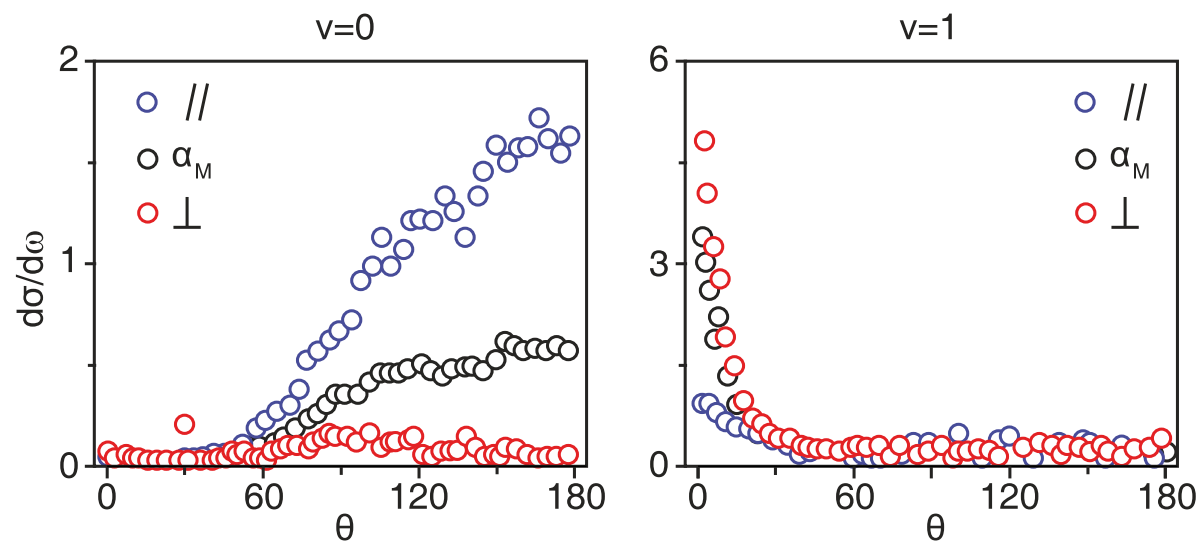

Figure 8: Polarization dependent angular distributions of $\mathrm{HCl} v=0$ (left) and $v=1$ (right) states formed after collisions of $\mathrm{CD}_{3} \mathrm{H}(v=1)+\mathrm{Cl}$. The angular distributions for three different polarizations of the IR laser used to excite the $\mathrm{CD}_{3} \mathrm{H}$. Parallel polarization results in a bond axis distribution aligned parallel to the relative velocity, with perpendicular polarization yielding an alignment of the $\mathrm{C}-\mathrm{H}$ bond axis perpendicular to the relative velocity. Magic angle polarization of the IR laser results in a practically isotropic distribution of the C-H bond axis. Adapted from Ref. [24]. 

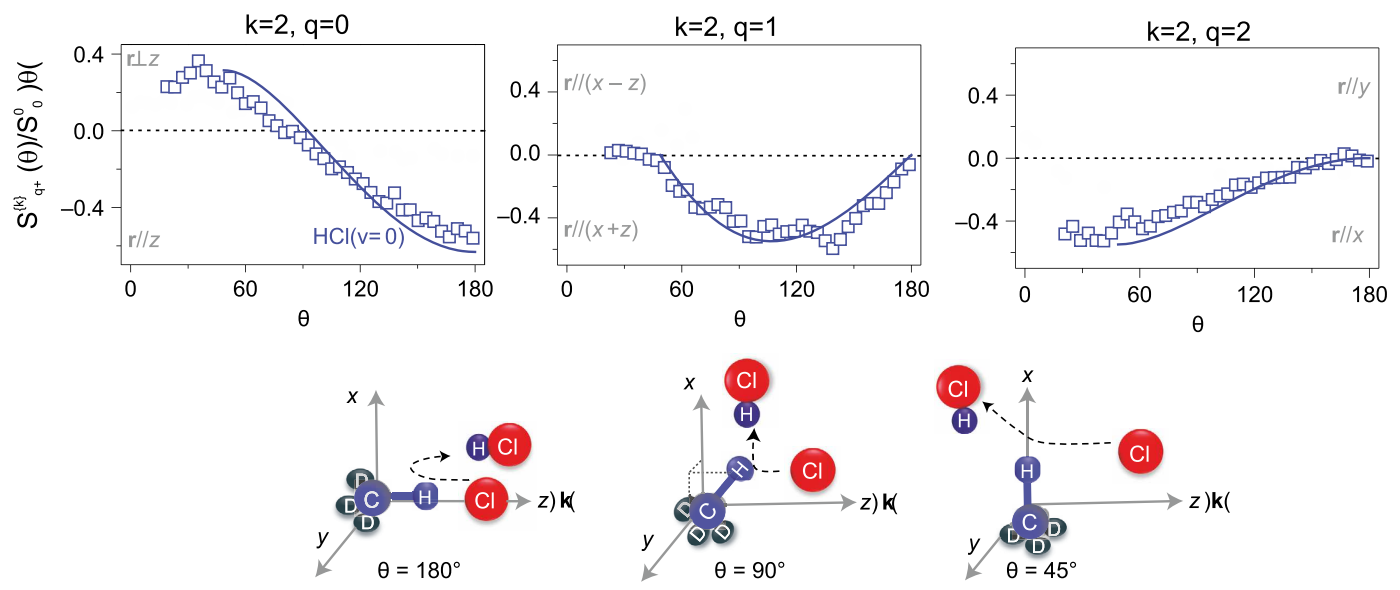

Figure 9: Experimentally determined $S_{q+}^{\{k\}}(\theta) / S_{0}^{0}(\theta)$ r-PDDCSs for collisions of aligned $\mathrm{CD}_{3} \mathrm{H}(v=1)$ with $\mathrm{Cl}$ for $k=2, q=0,1$ and 2 . The blue curves show the prediction from a line of centres model. A cartoon representation of the $S_{q+}^{\{k\}}(\theta) / S_{0}^{0}(\theta)$ values for three limiting scattering angles is shown in the lower panel. Adapted from Ref. [25]. 


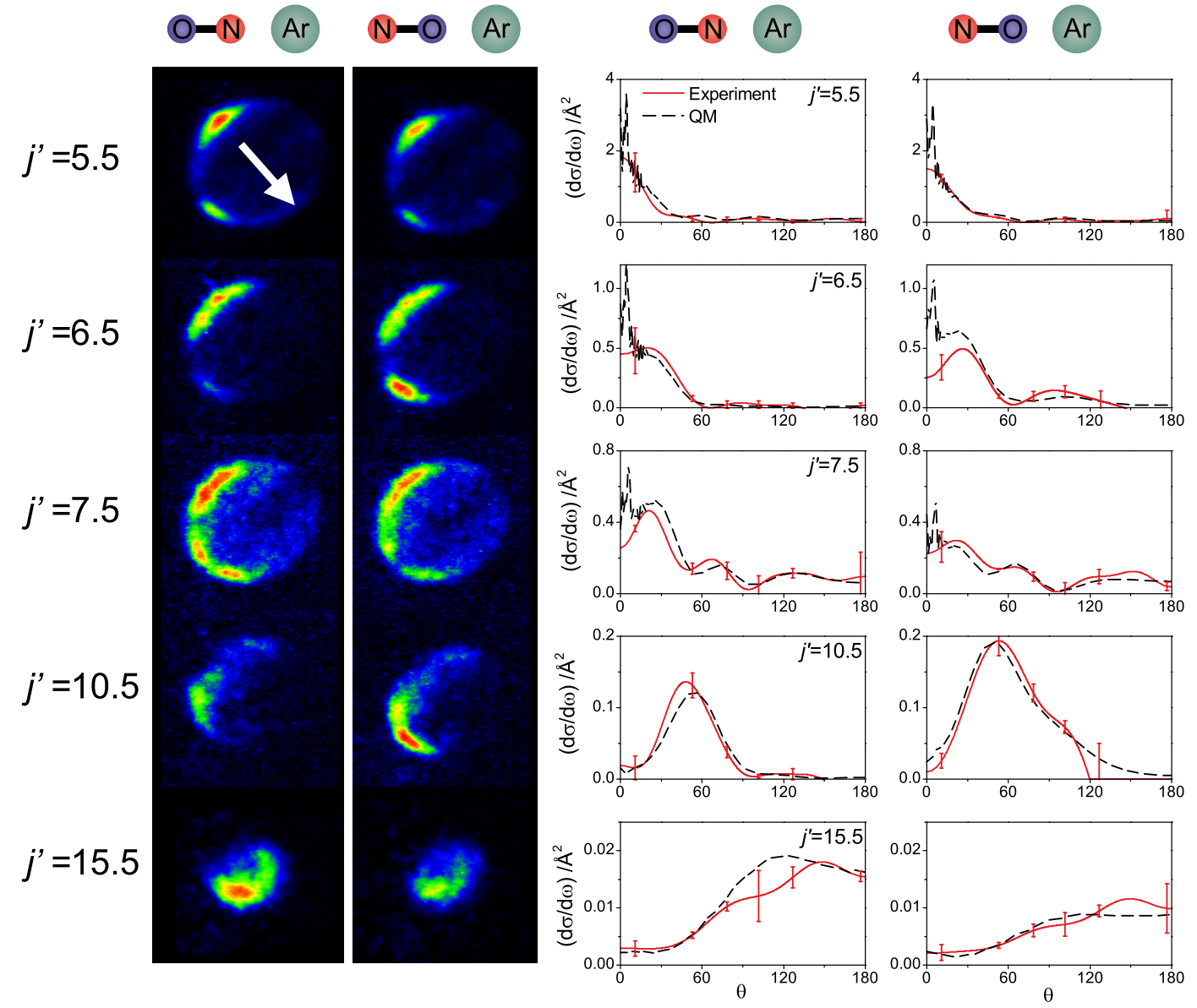

Figure 10: Experimental ion images for $\mathrm{N}$ - and O-end collisions of $\mathrm{NO}\left(X^{2} \Pi, v=0, j=1 / 2, \Omega=1 / 2\right)+$ Ar are shown in the first two columns. Experimentally determined differential cross sections (red lines) for the two bond axis orientations are compared with quantum mechanical calculations (black dashed line) are displayed in the third and fourth columns. The error bars represent the $95 \%$ confidence limits. Adapted from Ref. [73]. 

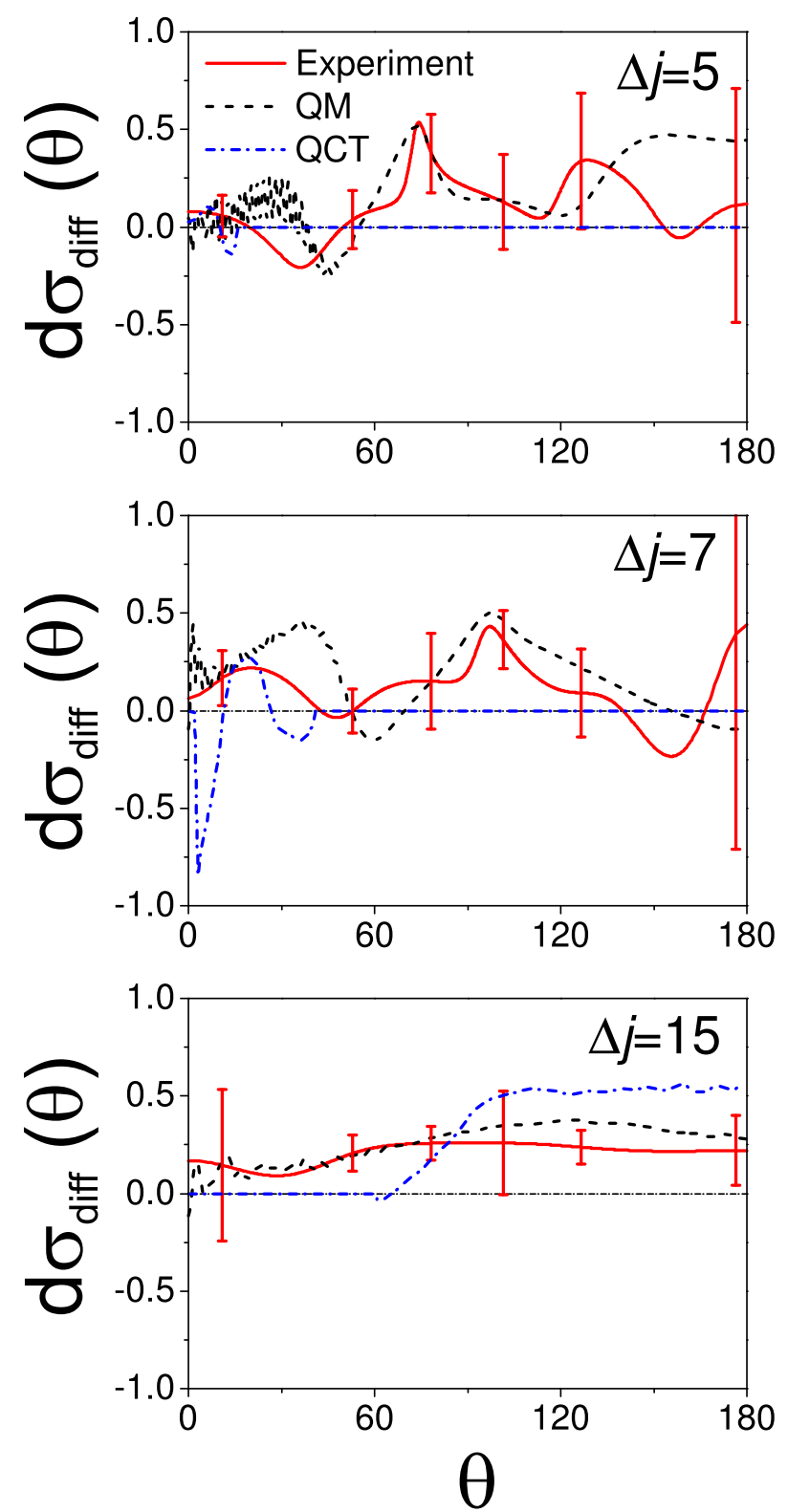

Figure 11: Normalized difference differential cross sections determined experimentally (red solid line), and from quantum mechanical (black dashed line) and quasi-classical trajectory calculations (blue dot dash line), for the inelastic scattering of $\operatorname{NO}\left(X^{2} \Pi, v=0, j=1 / 2, \Omega=1 / 2\right)$ by Ar. The error bars on the experimental data represent the $95 \%$ confidence limits. Adapted from Ref. [73]. 

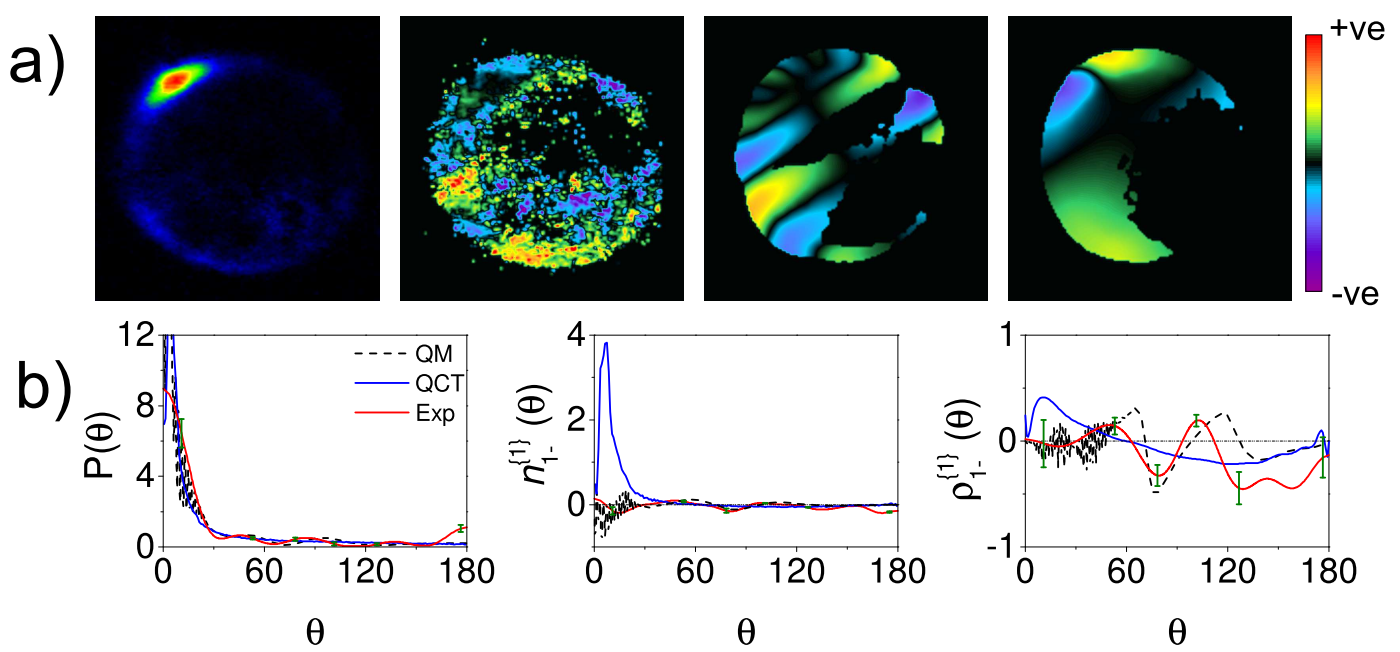

Figure 12: Panel a): Experimental images recorded using right $(\mathrm{R})$ and left $(\mathrm{L})$ handed circularly polarized light, presented as the sum $(\mathrm{L}+\mathrm{R})$ in the first column and normalized difference $(\mathrm{L}-\mathrm{R}) /(\mathrm{L}+\mathrm{R})$ in the second column. The data are for the inelastic scattering of $\operatorname{NO}\left(X^{2} \Pi, v=0, j=1 / 2, \Omega=1 / 2, f\right)$ by $\mathrm{Kr}$. The third and fourth columns display normalized difference images, simulated using quantum mechanical and quasi classical trajectory calculations respectively. Negative intensity indicates a propensity for counterclockwise rotation of the NO molecule if looked at from above. Panel b: A comparison of experimental (red line), quantum mechanical (black dashed line) and quasi-classical trajectory (blue line) calculated angular distributions (left column), normalized $j^{\prime}$-PDDCSs, (middle column) and renormalized $j^{\prime}$-PDDCSs (right column). The error bars on the experimental data represent the $95 \%$ confidence intervals. 